\title{
Numerical Investigations of the Benchmark Supercritical Wing in Transonic Flow
}

\author{
Pawel Chwalowski, ${ }^{*}$ Jennifer Heeg, ${ }^{\dagger}$ and Robert T. Biedron ${ }^{\ddagger}$ \\ NASA Langley Research Center, Hampton, VA 23681-2199
}

\begin{abstract}
This paper builds on the computational aeroelastic results published previously and generated in support of the second Aeroelastic Prediction Workshop for the NASA Benchmark Supercritical Wing (BSCW) configuration. The computational results are obtained using FUN3D, an unstructured grid Reynolds-Averaged Navier-Stokes solver developed at the NASA Langley Research Center. The analysis results show the effects of the temporal and spatial resolution, the coupling scheme between the flow and the structural solvers, and the initial excitation conditions on the numerical flutter onset. Depending on the free stream condition and the angle of attack, the above parameters do affect the flutter onset. Two conditions are analyzed: Mach 0.74 with angle of attack $0^{\circ}$ and Mach 0.85 with angle of attack $5^{\circ}$. The results are presented in the form of the damping values computed from the wing pitch angle response as a function of the dynamic pressure or in the form of dynamic pressure as a function of the Mach number.
\end{abstract}

\section{Nomenclature}

$\delta \quad$ structural displacement

$\hat{F}_{a} \quad$ generalized aerodynamic force

D damping matrix

I identity matrix

K stiffness matrix

M mass matrix

q dynamic pressure - psf

$\mathbf{x} \quad$ vector of generalized displacements and velocities

$\omega_{i} \quad$ natural frequency of $i^{\text {th }}$ mode

$\Phi \quad$ state-transition matrix

$\phi_{i}(\mathbf{x}) \quad \mathrm{i}^{\text {th }}$ mode shape
$\Theta \quad$ convolution integral of $[\Phi]$

$v_{n} \quad$ series coefficient for the representation of $\dot{\mathbf{x}}$

BDF Backward Differentiation Formula Scheme

BSCW Benchmark Supercritical Wing

CAE Computational Aeroelasticity

CFD Computational Fluid Dynamics

OTT Oscillating TurnTable

P-C Predictor Corrector

PAPA Pitch And Plunge Apparatus

TDT Transonic Dynamics Tunnel

TE Temporal Error

\section{Introduction}

$\mathrm{T}$ he second AIAA Aeroelastic Prediction Workshop (AePW-2) took place in conjunction with the AIAA SciTech 2016 Conference in San Diego, California. The computational aeroelasticity community was challenged to analyze one configuration at three conditions and to present their results at the workshop. The challenge cases utilized the NASA Benchmark Supercritical Wing (BSCW), which was first tested in the NASA Langley Transonic Dynamics Tunnel (TDT) in 1991. For this test, the wing was mounted on the TDT Pitch And Plunge Apparatus (PAPA) to obtain the flutter boundary at various Mach numbers and angles of attack for a two-degree of freedom (pitch and plunge) system. In 2000, the wing was tested again, this time on a TDT mounting mechanism called the Oscillating

*Senior Aerospace Engineer, Aeroelasticity Branch, Senior Member AIAA.

${ }^{\dagger}$ Senior Research Engineer, Aeroelasticity Branch, Associate Fellow AIAA.

${ }^{\ddagger}$ Research Engineer, Computational Aerosciences Branch, Member AIAA. 
Turntable (OTT). The purpose of the OTT tests was to measure aerodynamic response during sinusoidal (forced) pitch oscillation of the wing. The experimental data indicated that the BSCW exhibited a strong shock and boundary-layerinduced separated flow at a moderate angle of attack at transonic conditions. The computations of the transonic flow, in conjunction with the flutter boundary predictions, were the focus of the workshop. ${ }^{1}$

For code validations in general, the type of aerodynamic and/or aeroelastic phenomena to be analyzed is important since a validation process typically progresses from simpler to more challenging cases. For the AePW series, the approach being taken is to utilize existing experimental data sets in a building-block approach to incrementally validate targeted aspects of Computational Aeroelasticity (CAE) tools. Each block represents a component of a more complex nonlinear unsteady aeroelastic problem, isolating it such that the contributing physics can be thoroughly investigated. The challenge selected for the first AePW was the accurate prediction of unsteady aerodynamic phenomena on essentially rigid, geometrically simple models, with an additional foray into systems with weak coupling between the fluid and the structure. Results from this first workshop helped guide the direction of the second workshop, with analyses extending to include flutter prediction and therefore, increasingly complicated flow fields.

This paper builds on the computational aeroelastic results ${ }^{2}$ generated for the AePW-2 BSCW configuration using the NASA Langley-developed computational fluid dynamics (CFD) software FUN3D. ${ }^{3}$ While a previous publication concentrated on the overall summary of the computational results generated for the workshop, in this paper, we will be reporting on the variations of the numerical flutter boundary prediction due to the influences of: (1) temporal and spatial resolution, (2) the coupling scheme between the flow and the structural solvers, and (3) the initial excitation conditions. Two transonic conditions are examined: Mach 0.74 and Mach 0.85 , with angles of attack $0^{\circ}$ and $5^{\circ}$, respectively. The numerical results at Mach 0.74 are compared to the experimental data obtained during the PAPA test. Unfortunately, the experimental data for the PAPA flutter boundary at Mach 0.85 are not available for a similar comparison. In addition, because of the difficulty of predicting the flutter boundary at Mach 0.85 at an angle of attack $5^{\circ}$, this analysis effort was expanded to additional Mach numbers: 0.6, 0.7, 0.74, 0.8, 0.82 at that angle.

\section{BSCW Model and Test Cases}

The BSCW model, shown in Figure 1, has a simple, rectangular, 16- x 32-inch wing planform, with a NASA SC(2)-0414 airfoil. For both the PAPA and OTT tests, the model was mounted to a large splitter plate, sufficiently offset from the wind-tunnel wall (40 inches) to (1) place the wing closer to the tunnel centerline and (2) be outside the tunnel wall boundary layer. ${ }^{4}$ The wing was designed to be rigid, with the following structural frequencies for the combined installed wing and OTT mounting system: $24.1 \mathrm{~Hz}$ (spanwise first bending mode), $27.0 \mathrm{~Hz}$ (in-plane first bending mode), and $79.9 \mathrm{~Hz}$ (first torsion mode). When installed on the PAPA mount, the combined system frequencies were $3.33 \mathrm{~Hz}$ for the plunge mode and $5.20 \mathrm{~Hz}$ for the pitch mode. For instrumentation, the model has pressure ports at two chordwise rows at the $60 \%$ and $95 \%$ span locations, with 22 ports on the upper surface, 17 ports on the lower surface, and 1 port at the leading edge for each row. The BSCW/PAPA data consist of unsteady data at flutter points and averaged data on a rigidified apparatus at the flutter conditions. For this PAPA test, both the inboard row of pressures at the $60 \%$ span station and the outboard row at the $95 \%$ span station were populated with unsteady in situ pressure transducers. For the OTT test, however, only the inboard row at the $60 \%$ span station was populated with unsteady in situ pressure transducers.

The BSCW test case used in AePW-1 was Mach 0.85 at $5^{\circ}$ angle of attack. This proved to be more challenging than expected due to the shock-induced separated flow that dominated the upper surface and the aft portion of the lower surface at that condition. Using this information as a guide, two test cases just outside of the separated flow regime were emphasized for AePW-2. These are listed in Table 1. Steady and forced oscillation analyses were conducted at Mach $0.70,3^{\circ}$ angle of attack, and flutter analyses were conducted at Mach $0.74,0^{\circ}$ angle of attack. An optional Case \#3 at Mach $0.85,5^{\circ}$ angle of attack, which was the reanalysis of the AePW-1 case, was also encouraged to apply the higher fidelity tools. This optional case was divided into three separate subcases based on the type of dynamic data acquired: unforced unsteady, forced oscillation, and flutter.

In this paper, we are concentrating on the flutter boundary computations obtained using FUN3D of AePW-2 test Case \#2 and Case \#3c as highlighted in red in Table 1. In the following sections of this paper, the FUN3D computational results obtained for these cases are presented with emphasis on temporal and spatial convergence, the coupling scheme between the flow and the structural solvers, and the initial excitation conditions. 


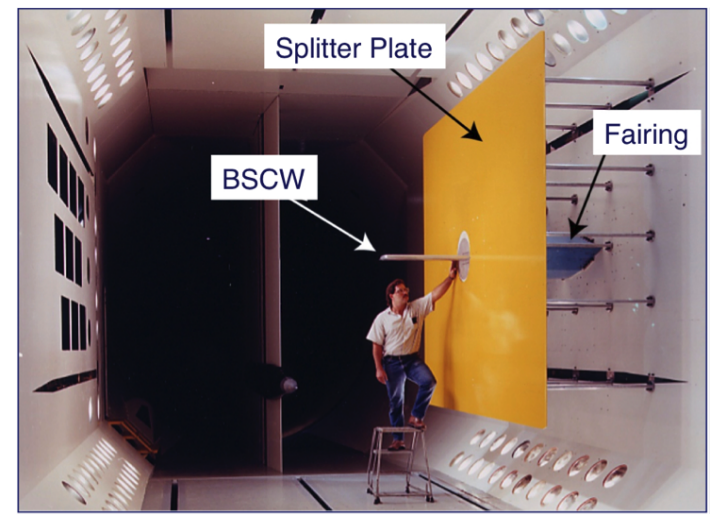

(a) Photograph of the BSCW model mounted on the OTT in the TDT.

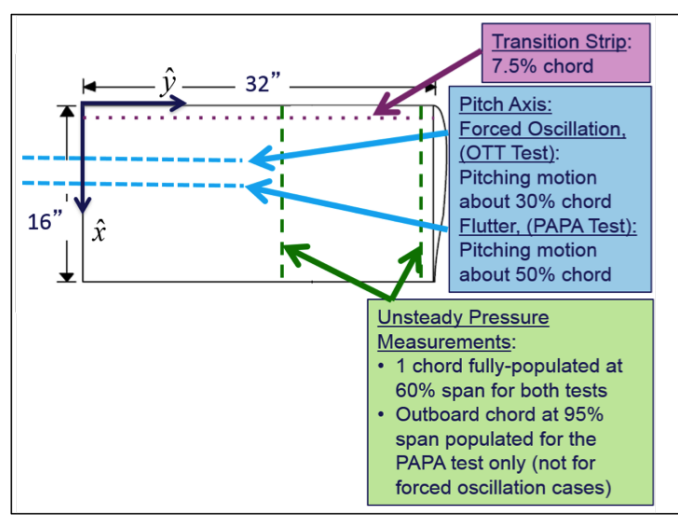

(b) BSCW geometry.

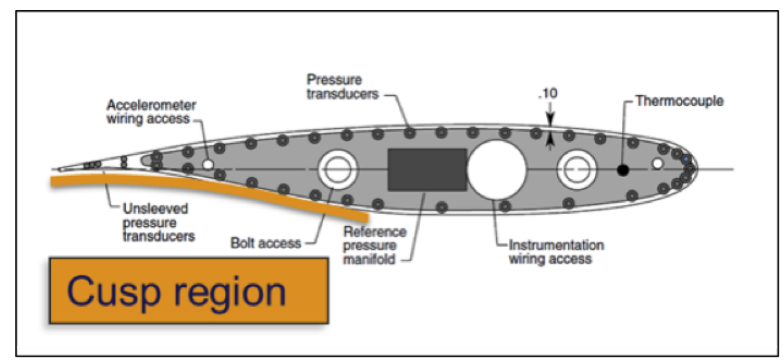

(c) Cross-sectional view of the SC(2)-0414 airfoil, with BSCW instrumentation.

Figure 1. BSCW Model.

Table 1. AePW-2 Workshop Test Cases.

\begin{tabular}{l|c|c|c|c|c}
\hline \hline & Case \#1 & Case \#2 & Optional Case \#3a & Optional Case \#3b & Optional Case \#3c \\
\hline Mach & 0.70 & 0.74 & 0.85 & 0.85 & 0.85 \\
\hline AoA & $3^{\circ}$ & $0^{\circ}$ & $5^{\circ}$ & $5^{\circ}$ & $5^{\circ}$ \\
\hline Dynamic & Forced & Flutter & Unforced & Forced & Flutter \\
Data Type & Oscillation & & Unsteady & Oscillation & \\
& $f=10 \mathrm{~Hz},|\theta|=1^{\circ}$ & & & $f=10 \mathrm{~Hz},|\theta|=1^{\circ}$ & \\
\hline \multirow{2}{*}{ Notes } & - Attached flow & - Flow state unknown & - Separated flow & - Separated flow & - Separated flow \\
& - OTT exp. data & - PAPA exp. data & - OTT exp. data & - OTT exp. data & - No exp. data \\
& - R-134a & - R-12 & - R-134a & - R-134a & - R-134a \\
\hline \hline
\end{tabular}

\section{Numerical Method}

\section{A. Grids}

For this study, unstructured grids consisting primarily of tetrahedra and prisms were generated using VGRID ${ }^{5}$ with input prepared using GridTool. ${ }^{6}$ The tetrahedral elements within the boundary layer were converted into prism elements using preprocessing options within the FUN3D software. Based on the AePW gridding guidelines, ${ }^{7}$ three grids belonging to the same family were then constructed, one with 3 million nodes, one with 9 million nodes, and one with 27 million nodes. These grids and the corresponding FUN3D solutions are referred to as 'coarse', 'medium' and 'fine', respectively, in this paper. In addition, a separate grid with 35 million nodes was constructed for use with the Delayed Detached Eddy Simulation (DDES) method. Called, Grid D, this grid was created to better resolve the wake region of the flow and to make the grid more isotropic in the separated flow region. The resulting four grid distributions for both the surface and the plane of symmetry are presented in Figure 2. 


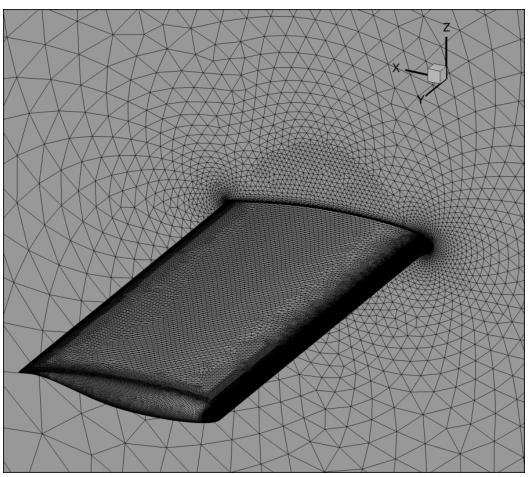

(a) Coarse Grid, Grid A, 3 Million Nodes.

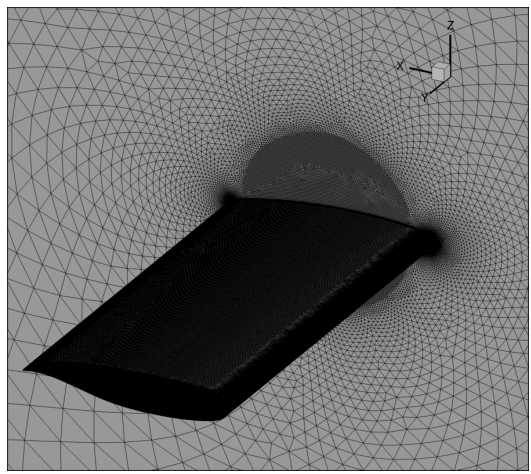

(c) Fine Grid, Grid C, 27 Million Nodes.

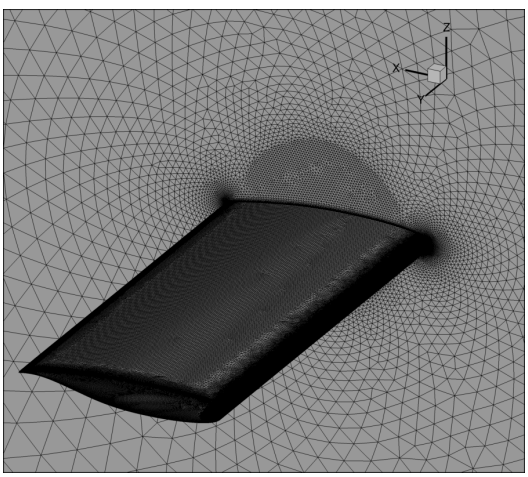

(b) Medium Grid, Grid B, 9 Million Nodes.

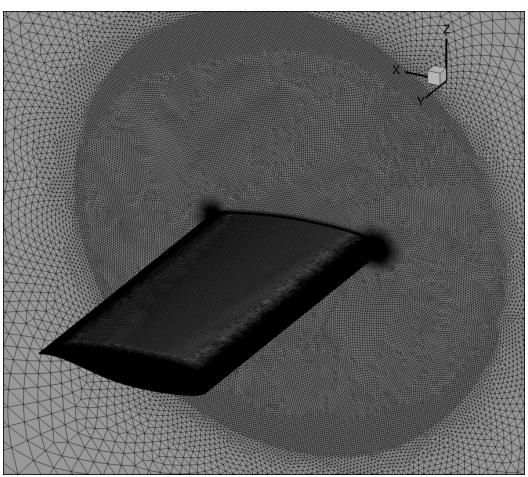

(d) Toward DDES, Grid D, 35 Million Nodes.

Figure 2. Coarse, Medium, Fine, and "Toward DDES" Grids.

\section{B. Rigid Steady-Flow Analysis - FUN3D}

Solutions to the Reynolds-Averaged Navier-Stokes (RANS) equations were computed using the FUN3D flow solver with turbulence closure obtained using the "standard" Spalart-Allmaras (SA) one-equation model. ${ }^{8,9}$ For the low transonic Mach number test cases selected for the BSCW analyses, oscillations in pressure fore and aft of the shock are relatively minor, so that the use of a flux limiter, with the accompanying adverse effects on solver convergence, is not essential; however, selected analyses were completed both with and without a flux limiter for comparison. Here, the flux limitation was accomplished with the Venkatakrishnan ${ }^{10}$ limiter, and inviscid fluxes were computed using the Roe scheme. ${ }^{11}$ For the asymptotically-steady cases, time integration was accomplished by an Euler implicit backward difference scheme, with local time stepping to accelerate convergence. Most of the cases in this study were run for about 5,000 iterations to achieve convergence of forces and moments to within $\pm 0.5 \%$ of the running average of the last 1,000 iterations.

\section{Dynamic Analysis - FUN3D}

Dynamic analyses of the BSCW configuration required unsteady-flow analysis. For unsteady-flow analysis, the FUN3D solver utilizes the dual-time-stepping method, which is widely used in CFD. This method involves adding a pseudo time derivative of the conserved variables to the physical time derivative that appears in the time-dependent Navier-Stokes equations, in much the same way that an artificial time term is often added to the steady Navier-Stokes equations to facilitate an iterative solution to a steady state. In the same manner used for a steady state solution with the pseudo time derivative vanishing as the iterations proceed within each time step toward the end of the iterative process, a solution to the original unsteady Navier-Stokes equations is obtained for that physical time step. Iteratively advancing each time step in pseudo time allows errors introduced by the (generally inexact) linearization of the nonlinear residual to be reduced to zero, assuming the iterations in pseudo time are fully converged. An additional advantage of the pseudo time term is that it enhances the diagonal dominance of the linear system, increasing robustness and allowing larger physical time steps to be taken than might otherwise be possible. For an infinitely large physical time step, the dual-time solver becomes identical to the steady-state solver, though of course, all time accuracy is lost. 
Aeroelastic analysis requires a grid deformation capability. The grid deformation in FUN3D is treated as a linear elasticity problem. In this approach, the grid points near the body can move significantly, while the points farther away may not move at all. In addition to the moving body capability, the analysis of the BSCW configuration requires a structural dynamics capability. For a dynamic aeroelastic analysis, FUN3D is capable of being loosely coupled with an external finite element solver, ${ }^{12}$ or in the case of the linear structural dynamics used in this study, an internal modal structural solver can be utilized. ${ }^{13}$ This modal solver is formulated and implemented in FUN3D in a manner similar to other NASA Langley aeroelastic codes $\left(\mathrm{CAP}-\mathrm{TSD}^{14}\right.$ and CFL3D $\left.{ }^{15}\right)$. For the BSCW computations presented here, the structural modes were obtained via a normal modes analysis (solution 103) with the Finite Element Model (FEM) solver MSC Nastran ${ }^{\mathrm{TM}} .{ }^{16}$ The modes were then interpolated to the surface mesh using the method developed by Samareh. ${ }^{17}$ The BSCW FEM was built by Heeg and described in reference 1 .

There are two methods implemented in FUN3D for coupling the flow solver with the structural solver. These methods are the Predictor-Corrector (P-C) scheme and the Backward Differentation Formula (BDF) scheme. Detailed descriptions of these methods are presented in the Appendix. Later in this paper, we present and compare the dynamic aeroelastic results obtained using both methods.

The BSCW dynamic analysis was performed in a multistep process. First, the steady CFD solution was obtained on the rigid body. In the case of a dynamic aeroelastic flutter solution, a static aeroelastic solution was then obtained by restarting the CFD analysis from the rigid-steady solution in a time-accurate mode ${ }^{18}$ with a structural modal solver, allowing the grid to deform. A high value of structural damping (0.99) was used so the structure could find its equilibrium position with respect to the mean flow before the dynamic response was started. Finally, the flutter solution was restarted from the static aeroelastic solution by setting the structural damping value to zero and providing an initial excitation 'kick' in the form of the generalized velocity. The effects of that initial excitation versus no excitation on the flutter solution are described later in this paper.

\section{AePW-2 Test Case \#2}

The second AePW-2 test case involved BSCW flutter prediction on the PAPA mount system at Mach 0.74 and $0^{\circ}$ angle of attack. As described in the previous section, for this analysis, the rigid steady solution was obtained first, followed by the static aeroelastic solution. The third step was to perform several dynamic aeroelastic FUN3D computations with different values of the dynamic pressure (q) in the vicinity of the experimental flutter dynamic pressure. The wing response in the form of the time varying pitch angle was then computed and used to calculate the damping ratio using a logarithmic decrement method. For a stable solution, the damping ratio is greater than zero, and for an unstable solution, the damping ratio is less than zero. The damping ratio and the dynamic pressure were interpolated, and at zero damping ratio, the dynamic pressure was considered to be the flutter dynamic pressure. Once this flutter dynamic pressure was identified, the corresponding flutter frequency was determined via an interpolation of the frequencies. For this analysis process, dynamic solutions were obtained for the medium grid at several dynamic pressures, ranging from 1 to 169 psf. The high end of this range, at $169 \mathrm{psf}$, was chosen because it is the experimentally-obtained flutter q. The resulting computed damping and frequencies are presented in Figures $3 \mathrm{a}$ and b, respectively. An averaged dynamic pressure of $151.7 \mathrm{psf}$ was declared to be the computationally-obtained flutter dynamic pressure, a value that is approximately $10 \%$ below the experimental flutter dynamic pressure. The numerical flutter frequency for all three grids was determined to be approximately $4.2 \mathrm{~Hz}$.

\section{A. Temporal and Spatial Effects}

Next, the temporal and spatial effects on the computationally-obtained flutter dynamic pressure are examined. Table 2 shows the time-step sizes and criteria used in this analysis. As previously noted, the highest structural modal frequency for the BSCW model on the PAPA mount system was for the pitch mode at approximately $5 \mathrm{~Hz}$. The first column in the table shows the number of samples per $5 \mathrm{~Hz}$ cycle that were considered in the analysis. Columns 2 and 3 show the corresponding physical and nondimensional time step sizes used in the analysis. While it is recognized that the nondimensional time steps of DT $=12$ and DT $=24$ are too large, it was decided to conduct FUN3D analyses with these values for comparison purposes. Finally, column 4 shows the number of cycles per characteristic time scale of the free stream flow traveling across the airfoil.

In the previous analysis effort, ${ }^{2}$ it was shown that the proper selection of both the time-step size and number of subiterations in the dual-time stepping scheme is very important in flutter boundary prediction. Figure $4 \mathrm{a}$ shows the generalized displacements obtained for the plunge and pitch modes (modes 1 and 2) when FUN3D was executed with three levels of nondimensional time-step sizes (DT) corresponding to dimensional time-step sizes of 0.0002, 0.002, 


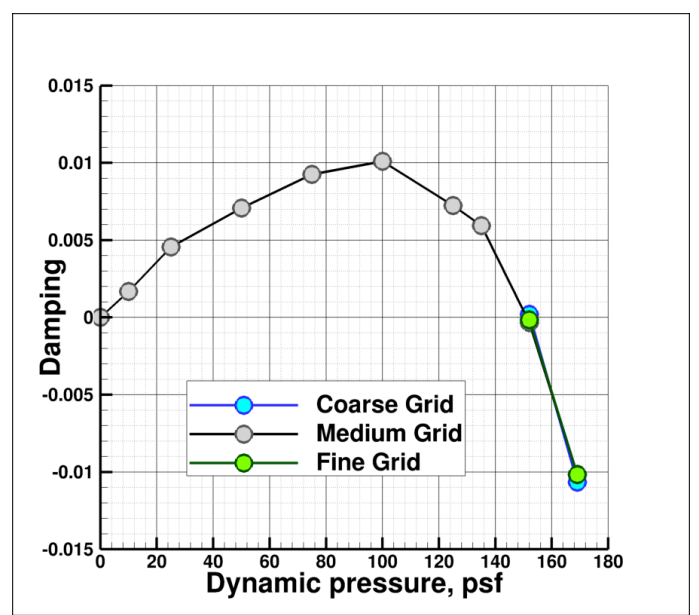

(a) Damping Value as a Function of Dynamic Pressure.

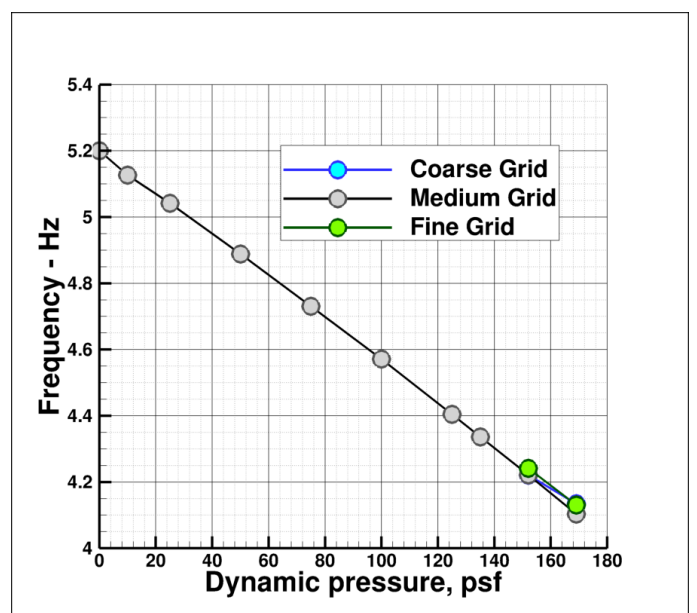

(b) Frequency as a Function of Dynamic Pressure.

Figure 3. Case \#2: Search for Flutter Dynamic Pressure and Flutter Frequency, Mach 0.74, $\operatorname{Re}_{c}=4.45 * 10^{6}, \alpha=0^{\circ}$.

and 0.004 seconds. At these time steps, the solution behavior changes from stable ( $\mathrm{dt}=0.004$ seconds - green curve), to slightly unstable $(\mathrm{dt}=0.002$ seconds - black curve), to unstable $(\mathrm{dt}=0.0002$ seconds - red curve). All the solutions were obtained by running FUN3D on a coarse grid with 15 subiterations at each physical time step. Figure $4 \mathrm{~b}$ shows the continuation of the temporal convergence study, where in addition to the unstable solution with the 0.0002 seconds time step (red curve, which is not visible because it is very close to the blue curve), two additional solutions were added. One was obtained with the same $\mathrm{dt}=0.0002$ seconds, but with 1000 subiterations (blue curve). The second solution was obtained by reducing the time-step size to 0.00002 seconds but the number of subiterations was kept at 15 (orange curve). These three solutions produced nearly identical results. Therefore, it is concluded that the FUN3D solution on a coarse grid obtained with a time-step size of 0.0002 seconds and 15 subiterations (or more) is sufficient for Case \#2 flutter prediction. Similar analyses were performed running FUN3D on medium and fine grids. Those results showed that a minimum of 25 subiterations with the time step of 0.0002 seconds are needed for Case \#2 flutter prediction. Further investigation of these solutions showed that different convergence levels at the subiteration level were obtained during wing-motion cycle. Typically, when the actual angle of attack was at the highest value during the cycle, more subiterations were needed to drop the residuals to the satisfactory level than when the angle of attack was small.

In the previous work discussed in reference 2, it was also pointed out that the FUN3D solver employs a temporal error (TE) control parameter to provide a residual-cutoff region for the solution to stop at the subiteration level and to proceed to the next physical time step. This cutoff occurs when the residuals drop below the product of that parameter and an estimate of the temporal error. In this study, for all but the smallest time-step analysis, the maximum of 200 subiterations was specified, with a temporal error control parameter of either 0.05 (5\%) or 0.1 (10\%). Number of subiterations was kept at 15 for the results obtained with the physical time-step size of 0.00002 seconds due to the computational expense. On average, the residuals drop about seven and eight orders of magnitude at the subiteration level with the temporal error control parameter set to $0.05(5 \%)$ or $0.1(10 \%)$, respectively, with the dimensional timestep size set to 0.0002 . However, the residuals drop only four orders of magnitude with 15 subiterations with the same time step. The resulting responses of the wing, in the form of the pitch angle, are plotted for different grid and temporal resolutions. For simplicity, only the analyses at dynamic pressures of 152 psf and 169 psf are shown.

Figure 5 shows the pitch angle response in time when FUN3D was executed with three levels of grid resolution (coarse, medium, and fine) and two values of temporal error controller $(5 \%$ and $10 \%)$ at the dynamic pressure of $\mathrm{q}=$ $169 \mathrm{psf}$ and the nondimensional time-step size of DT $=1$. The results show that there is a very small sensitivity to the grid resolution and subiteration-level convergence on the wing response when the time-step size is kept at DT $=1$. The influence of different time-step sizes is shown in Figure 6, which shows the wing pitch angle response at the three grid resolutions (coarse, medium, and fine) for all of the time-step sizes considered in this study. From these plots, it is clear that the time-step size is an important parameter in the flutter calculations.

Another way of looking at these results is to plot the computed damping values as a function of the dynamic pressure. Figure 7 shows the computed damping values for the coarse, medium, and fine grids using nondimensional time-step sizes of $0.1,1,12$, and 24 and at two temporal error convergence criteria (5\% and 10\%) at two dynamic 


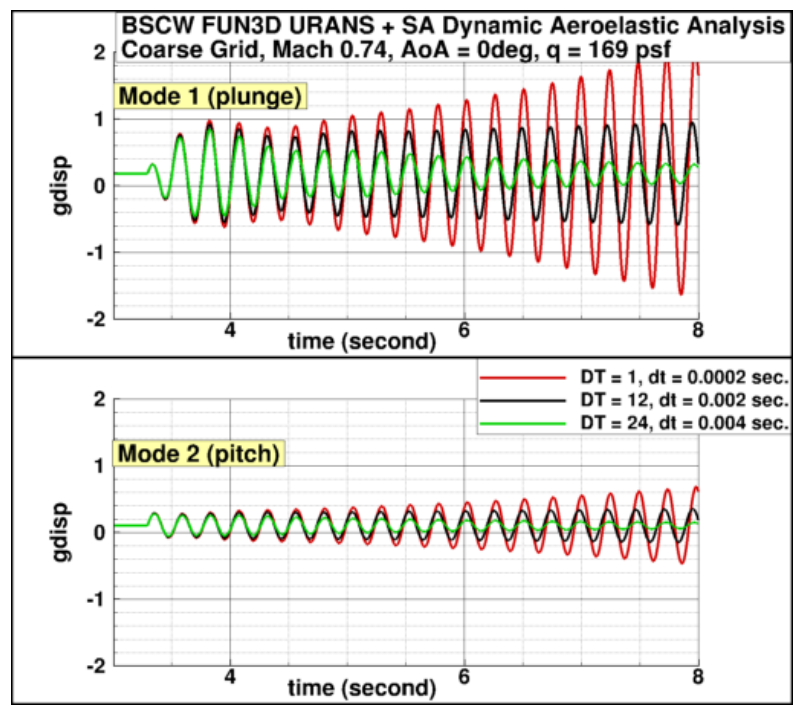

(a) Computed Generalized Displacements at Three Time-Step Levels (DT $=1,12,24)$ and 15 subiterations.

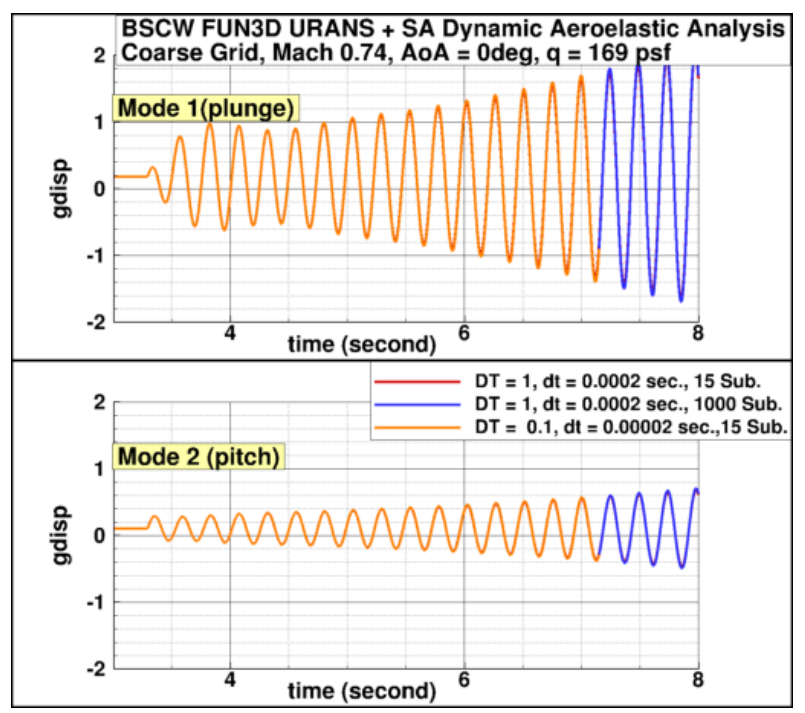

(b) Computed Generalized Displacements at Two Time-Step Levels (DT $=1,0.1$ ) and 15 and 1000 subiterations.

Figure 4. Time History of the Generalized Displacements at Various Time-Step Sizes, BSCW, Mach 0.74, q = 169 psf, $\operatorname{Re}_{c}=$ $4.45 * 10^{6}, \alpha=0^{\circ}$.

Table 2. BSCW Analysis, Temporal Parameters, Mach 0.74, $\alpha=0^{\circ}$.

\begin{tabular}{c|c|c|c}
\hline \hline Samples / 5 Hz & Physical time step dt (sec) & Nondimensional time step (DT) & Cycles per characteristic time \\
\hline 50 & 0.004 & $24.38(\mathrm{DT}=24)$ & $\sim 1$ \\
100 & 0.002 & $12.19(\mathrm{DT}=12)$ & $\sim 2$ \\
1000 & 0.0002 & $1.219(\mathrm{DT}=1)$ & $\sim 20$ \\
10000 & 0.00002 & $0.1219(\mathrm{DT}=0.1)$ & $\sim 200$ \\
\hline \hline
\end{tabular}

pressures (152 psf and $169 \mathrm{psf}$ ). Careful examination of these plots shows that the analyses with the finer grids, smaller time steps, and smaller value of the temporal error criteria push the flutter boundary away from the experimental flutter boundary dynamic pressure of $\mathrm{q}=169 \mathrm{psf}$. At the same time, the medium-grid solutions appear to be the outliers in these analyses and require further investigation.

\section{B. Flow and Structural Solvers Coupling Algorithms}

One of the most frequently asked questions during the AePW-2 workshop was about the influence of the fluid/structure coupling schemes in CAE software on the flutter prediction. This section addresses the results obtained during this analysis effort for the two methods available in FUN3D: a Predictor-Corrector (P-C) and a Backward Differentiation Formula (BDF) coupling schemes. The details of these two schemes are described in the Appendix. As pointed out in the Appendix and repeated here, historically, the coupling between the flow solution and the structural solution has been one way. With each physical time step, the flow equations are solved first, followed by the structural-dynamics equations with the corrector bringing in the latest flow solution in the physical time step. Within this physical time step, the flow equations are never solved again using the latest structural solution. The BDF scheme in FUN3D was implemented to unify the time advancement for both fluid and structures and potentially provide a tighter coupling between the two. When the BDF scheme is used to advance the structural equations, the subiteration counter is common to the same subiteration counter used to advance the flow equations in the dual-time stepping scheme. In FUN3D, the termination of the subiteration loop due to temporal error convergence, is based on the requirements of the flow equations, and not the structural equations.

Figure 8 shows the generalized displacements for the plunge and pitch modes, respectively, when FUN3D was executed in five different ways. First, FUN3D was run in a traditional way using the P-C scheme with 200 subiterations. 


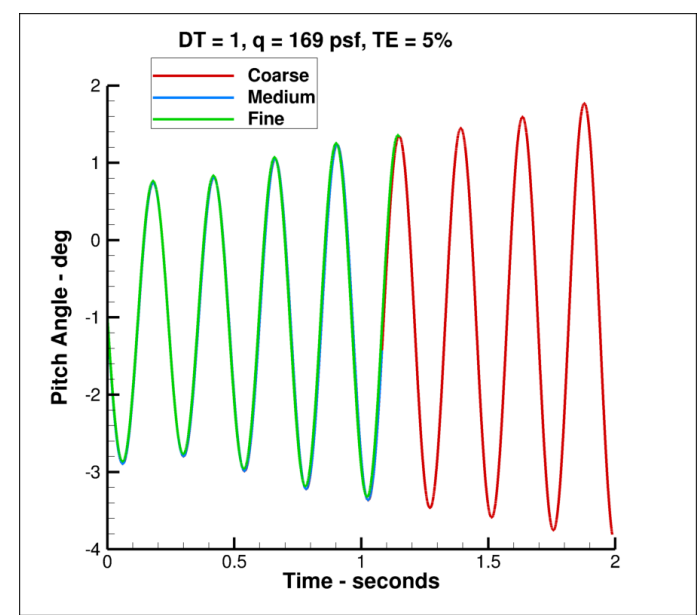

(a) Coarse, Medium, and Fine Grids Effect, Temporal Error $=5 \%$.

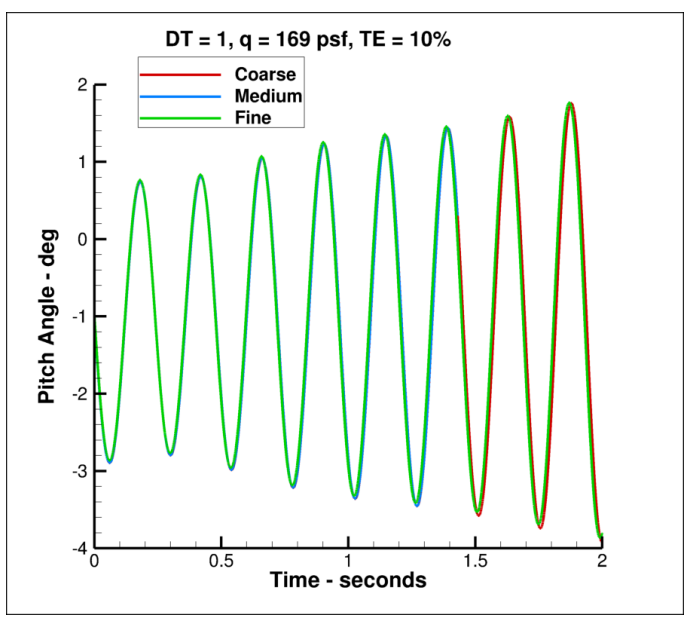

(b) Coarse, Medium, and Fine Grids Effect, Temporal Error $=10 \%$.

Figure 5. Case \#2: Grid Resolution Effects on the Wing Pitch Angle Response, DT = 1, q = 169 psf, Mach 0.74, $\alpha=0^{\circ}$, TE $=5 \%$, and $10 \%$.

This means that the coupling between the fluid and the structure occurred only once at the end of each physical time step. This solution is labeled as 'P-C 200sub' and is represented by the blue line. Next, FUN3D was run using the BDF scheme with two fluid-structural couplings and 100 subiterations per each coupling within one physical time step. This solution is labeled as 'BDF $2 \times 100$ sub' and is represented by the green line. FUN3D was then run using the BDF scheme with three fluid-structural couplings and 67 subiterations per coupling within one physical time step. This solution is labeled as 'BDF $3 \times 67$ sub' and is represented by a dark purple line. Next, FUN3D was run using the BDF scheme with 10 fluid-structural couplings and 20 subiterations per coupling within one physical time step. This solution is labeled as 'BDF 10x20sub' and is represented by the orange line. Finally, FUN3D was run using the $\mathrm{P}-\mathrm{C}$ scheme with two fluid-structural couplings and 100 subiterations per coupling within one physical time step. This solution is labeled as 'PC 2x100sub' and is represented by a dashed black line. The results show that for the Mach 0.74 , $\alpha=0^{\circ}$ case, there is no significant difference between the P-C and BDF schemes for the flutter prediction. However, it is recognized that this case represents a simple aerodynamic condition with a two-degree of freedom system. The $\mathrm{P}-\mathrm{C}$ and BDF schemes need to be tested on a more challenging test case.

\section{Initial Perturbation Effect and Structural Damping Influence}

As mentioned in Section IIIc, the typical flutter calculation in FUN3D requires an initial excitation 'kick' at the start of the dynamic aeroelastic simulation. This kick can be applied in the form of either (generalized) velocity or force. The magnitude of the kick is somewhat arbitrary; however, it cannot be so large that it causes large grid deformation and possible solution failure. A large kick in the pitch degree of freedom may also change the aerodynamic state of the flow, leading to incorrect flutter prediction for systems assumed to be subject to small perturbations in free stream conditions. On the other hand, a kick that is too small will result in the solution taking a very long time to develop. However, in general, an initial excitation kick might not be required to obtain a flutter solution. Some participants of the AePW-2 workshop chose to execute the dynamic solution directly from the rigid-steady solution. ${ }^{19}$ This is possible because there is a significant transient as the system moves from the rigid shape and position to the aeroelastic equilibrium shape and position. By shape and position here, we mean wing twist and elevation (pitch and plunge displacements). During this analysis effort, FUN3D was executed in a similar way. Figure 9a shows the damping values computed at dynamic pressures of 152 psf and 169 psf when the FUN3D dynamic solutions were restarted from the rigid-steady solution. The results are very similar to those presented in Figure 7.

Although Dansberry ${ }^{20}$ estimated that the experimental structural damping ratio of the BSCW on the PAPA mount could be 0.002 , most workshop participants used a zero value damping ratio in their analyses. FUN3D was executed on a fine grid with input damping ratio values of both zero and 0.002 . Figure $9 \mathrm{~b}$ shows that an input damping ratio of 0.002 shifts the damping vs. dynamic pressure curve in the vertical direction by 0.002 , indicating a linear effect of structural damping. Incorporating the measured structural damping ratio moves the flutter boundary toward the experimental flutter boundary by about 5 psf. 


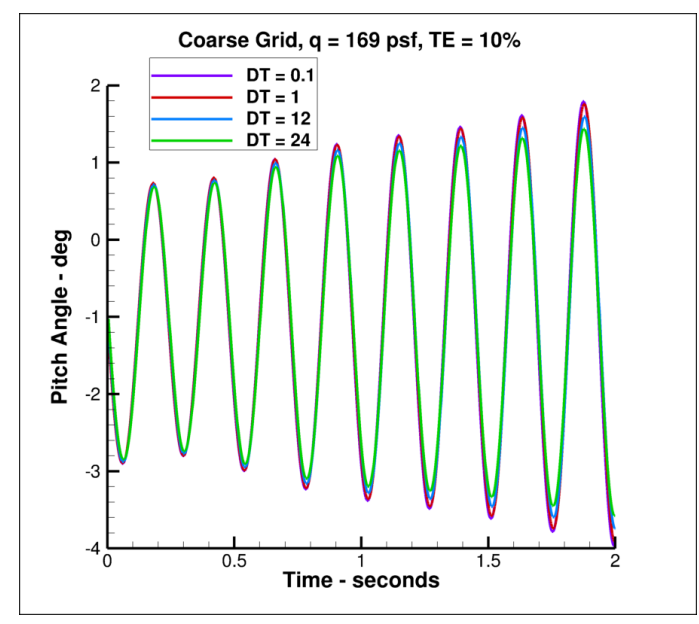

(a) Coarse Grid.

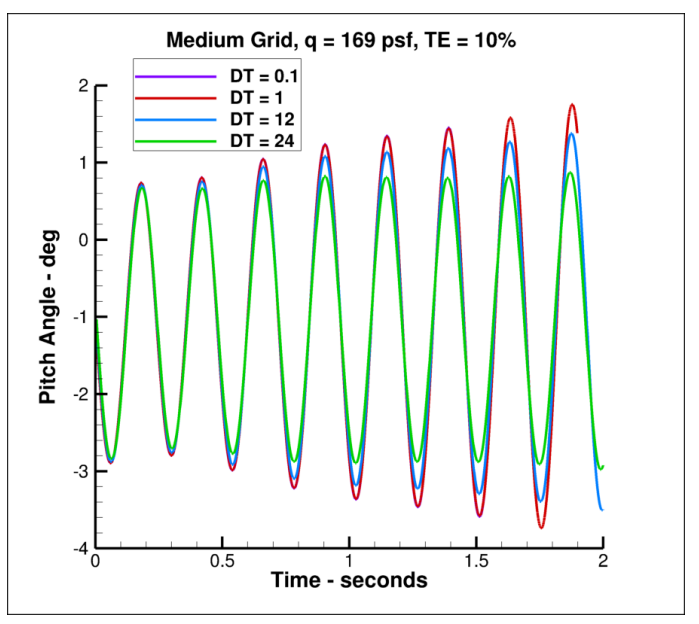

(b) Medium Grid.

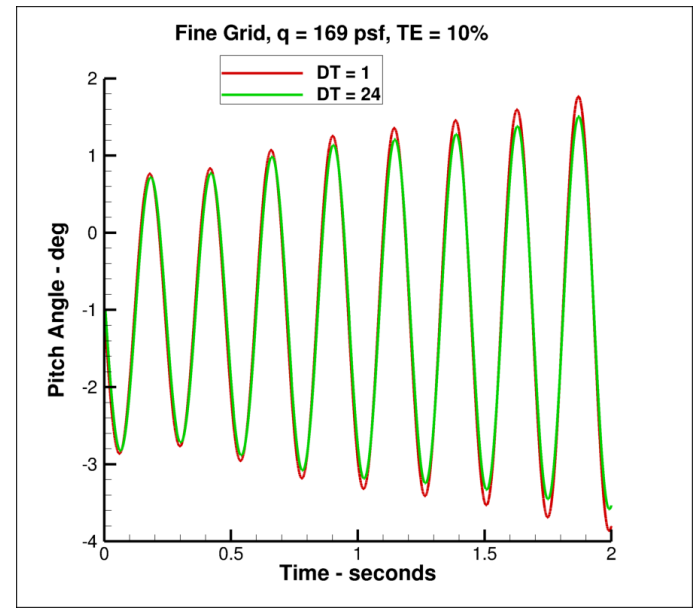

(c) Fine Grid.

Figure 6. Case \#2: Pitch Angle for Coarse, Medium, and Fine Grids, DT = 1, 12, and 24, Mach 0.74, $q=169$ psf, $\alpha=0^{\circ}$, $\mathrm{TE}=10 \%$.

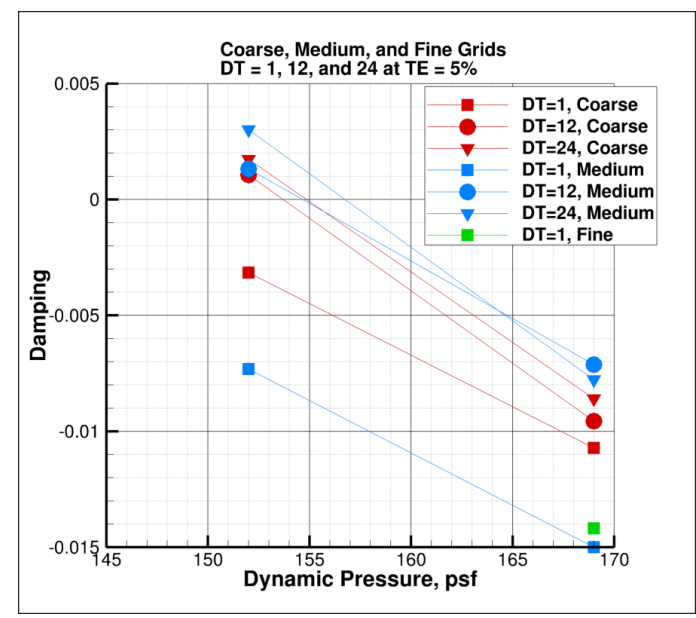

(a) Coarse, Medium, and Fine Grids Effect, DT=1, 12, and $24, \mathrm{TE}=5 \%$.

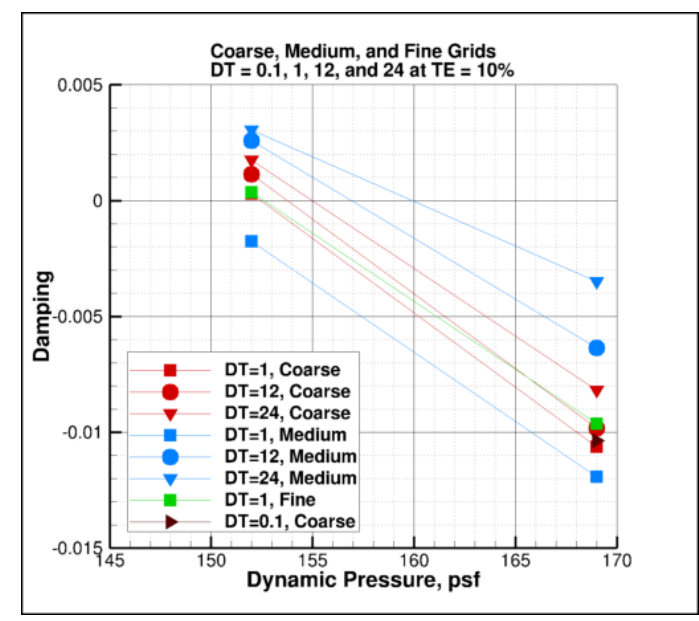

(b) Coarse, Medium, and Fine Grids Effect, DT=0.1, 1, 12, and $24, \mathrm{TE}=10 \%$.

Figure 7. Case \#2: Grid Resolution and Time Resolution Effects on the Damping Value, $q=152$ and 169 psf, Mach 0.74, $\alpha=0^{\circ}, \mathbf{T E}=\mathbf{5 \%}$ and $10 \%$. 


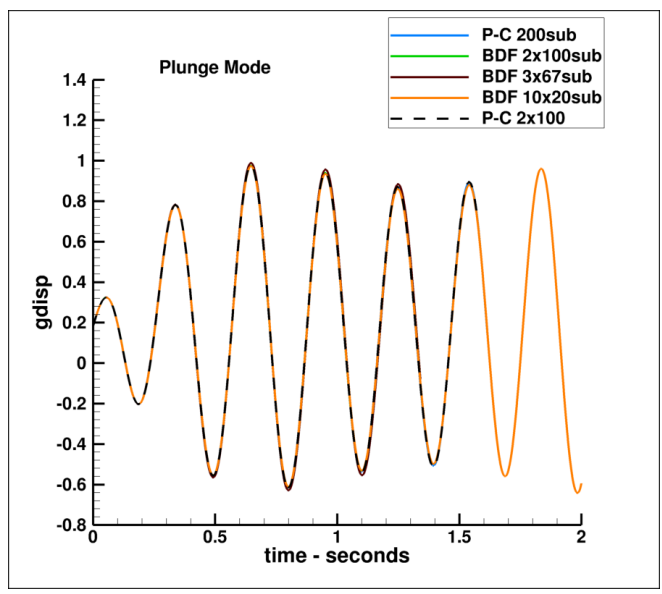

(a) Plunge Mode.

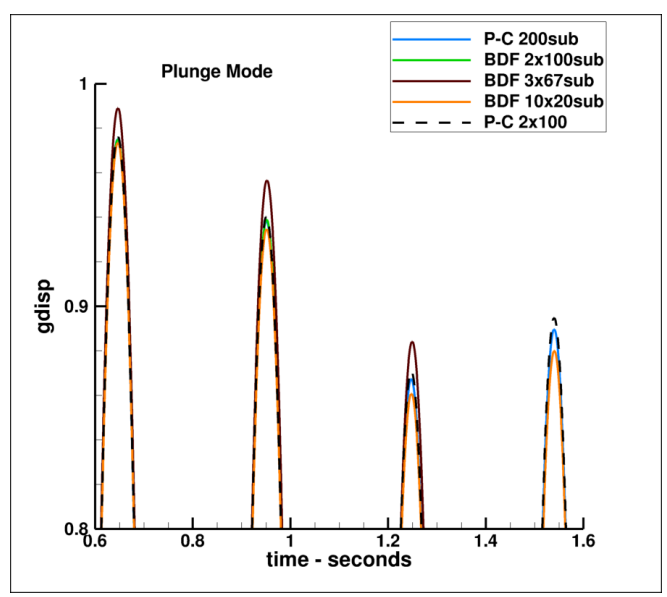

(c) Plunge Mode - Zoom in.

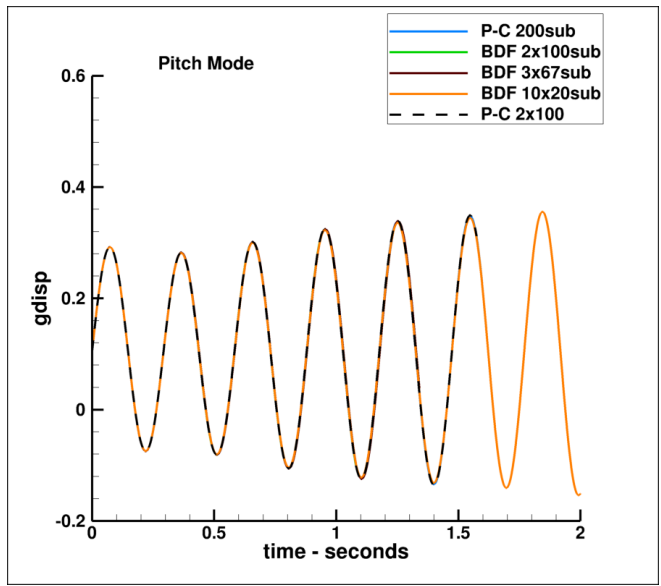

(b) Pitch Mode.

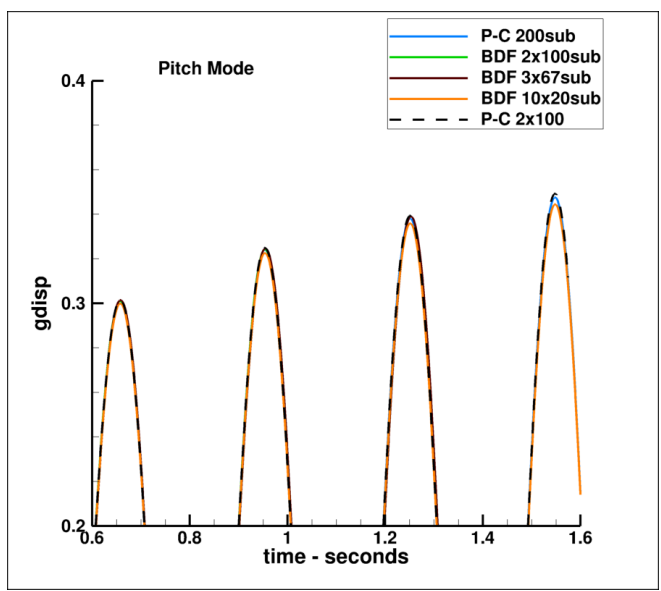

(d) Pitch Mode - Zoom in.

Figure 8. Generalized Displacements for the BSCW Plunge and Pitch Modes, Predictor-Corrector (labeled as P-C) and Backward-Difference (labeled as BDF) Coupling Schemes, Coarse Grid, $q=169$ psf, Mach 0.74, $\alpha=0^{\circ}$.

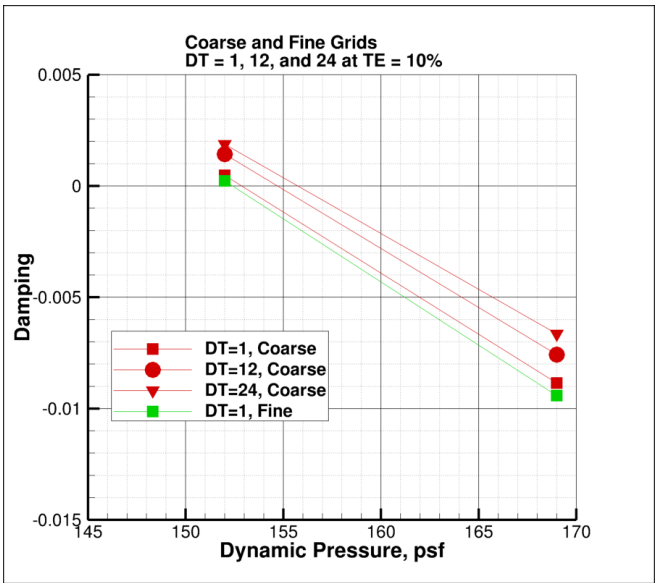

(a) Coarse and Fine Grid Solutions Obtained when the Dynamic Solution Was Restarted from the Rigid-Steady Solution.

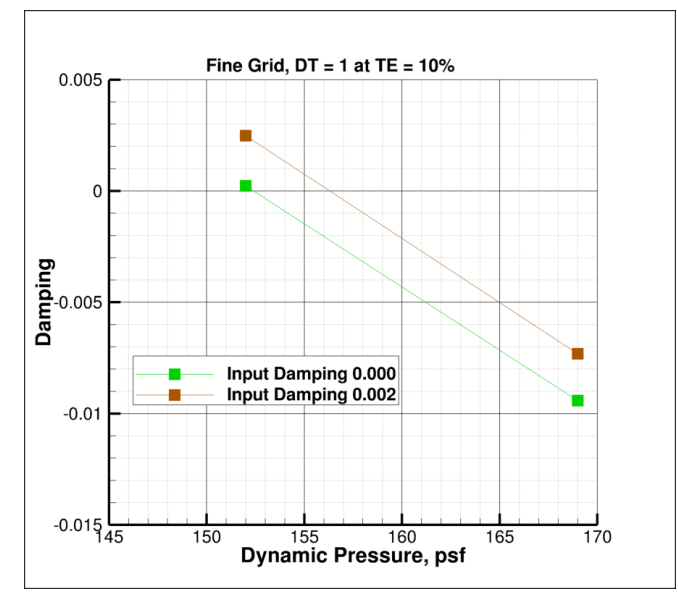

(b) Influence of the Input Damping Ratio Values.

Figure 9. Effects of No Initial Perturbation Effect and Damping Ratio Value, $q=152$ psf and 169 psf, Mach 0.74, $\alpha=0^{\circ}$, $\mathrm{TE}=10 \%$. 


\section{AePW-2 Test Case \#3c}

AePW-2 test Case \#3 was an optional workshop case that was a carryover from AePW-1. This case focused on the BSCW model at Mach $0.85,5^{\circ}$ angle of attack. The AePW-1 results showed significant scatter in the data reported by the different analysis teams. Based on these AePW-1 results and recommendations from the community, this case was split into three parts. The first subcase, designated Case \#3a, assessed the rigid-steady versus rigid-unsteady flow calculations in the presence of the shock-induced separated flow, which dominates the upper surface and the aft portion of the lower surface. The second subcase, designated Case \#3b, computed the flow around the wing undergoing forced oscillation at $10 \mathrm{~Hz}$. For both of these cases, some experimental data are available. The third and the most challenging subcase, designated Case \#3c, was the flutter onset prediction at this condition. Unfortunately, experimental data do not exist for this case, so it was considered to be a 'blind' test case. This third optional test case was the focus of the reanalysis effort presented here. Temporal convergence analyses were not conducted for this case and the FUN3D was executed with the nondimensional time-step size of DT $=1$.

The process for finding the flutter boundary for Case \#3c is the same as that outlined and used for Case \#2. Figure 10a provides a summary of the predicted results from the AePW-2 efforts ${ }^{2}$ and graphically demonstrates the issues and various solutions associated with computation of the flutter boundary for this case. Depending on the grid resolution and the scheme used, the predicted flutter dynamic pressure varies over a range of $280 \mathrm{psf}$ at Mach 0.85 . It is because of this very wide range of flutter prediction that we recommended further analysis.

From analyses performed in support of AePW-2, it was determined that the application of a limiter in the solution process at Mach 0.85 and $5^{\circ}$ angle of attack shifts the predicted shock location aft on the upper surface of the wing, affecting the size of the separated region behind the shock. ${ }^{2}$ In addition, the grid resolution affects both shock strength and spatial position. As shown in Figure 10a, the range of the predicted dynamic pressure, between the coarse and fine grid solutions with a limiter, is nearly 200 psf. On the other hand, the corresponding range for the solutions without the limiter is only about $100 \mathrm{psf}$. In addition, a DDES solution on a fine grid produced a flutter boundary point in between the coarse- and fine-grid solutions. This very wide range of predictions prompted an attempt to calculate the flutter boundary over a wide range of Mach numbers from 0.6 to 0.85 at $5^{\circ}$ angle of attack.

The calculation at Mach 0.82 on a coarse grid produced a flutter boundary point at $200 \mathrm{psf}$ while the coarse grid calculations at Mach numbers of 0.8 and 0.74 did not establish flutter boundary predictions at all. All solutions were always unstable, even with a low dynamic pressure of $25 \mathrm{psf}$. On the other hand, the calculations at Mach numbers of 0.6 and 0.7 predicted a nearly identical flutter boundary dynamic pressure of about 110 psf. The calculations at Mach 0.80 and Mach 0.74 were repeated using Grid D with the DDES turned on and off. However, these calculations produced nearly the same flutter boundary prediction, with the flutter dynamic pressures at Mach 0.8 and Mach 0.74 computed to be 55 psf and 68 psf, respectively.

The results from the current analysis effort are presented in Figure 10b. The brown-dashed curve shows the flutter boundary prediction using Grid D only with the SA turbulence model. These results show some refinement in the flutter boundary prediction at almost all Mach numbers, probably due to a better grid resolution in the wake region. Additional points were also added at Mach 0.85 from the analysis using the medium grid. These medium grid results, both with and without a limiter, match the trend with the coarse and fine grid results. At the same time, more results at different dynamic pressures were obtained, which improved the interpolation process to find the dynamic pressure at the zero damping value. Considering the results for Grid D only with the SA turbulence model, the flutter at Mach 0.85 is predicted at the dynamic pressure $\mathrm{q}=437 \mathrm{psf}$. The corresponding prediction for the fine grid without a limiter is at $\mathrm{q}=394 \mathrm{psf}$, while the preliminary prediction of flutter dynamic pressure for Grid D with the DDES solution is at $\mathrm{q}=428 \mathrm{psf}$.

Figures $11,12,13$, and 14 show visually the flow complexity during one cycle of the solution development. The flow conditions are at Mach 0.70, 0.80, 0.82, 0.85. In these figures, the '(a)' subplot shows the location during the pitch-angle cycle where the data were extracted. The results are plotted at the dynamic pressures closest to the flutter dynamic pressure at which computations were performed. These results consist of Mach number contours on the plane of symmetry (or root of the wing) and skin friction on the wing surface. The skin friction is shown as positive (brown color) and negative (light blue color) values. The five points are separated by 200 physical time steps. These figures demonstrate the development of the separated flow region behind the shock. Clearly, the flow in the transonic bucket region is characterized by the mixed separated and attached flow. The separated flow region increases in size with the increasing Mach number. The shock-motion direction also changes. At Mach 0.7, when the wing pitches up, the shock moves aft on the wing, while at Mach 0.85 the shock moves forward. Interestingly, the frequency of the pitch-angle oscillation near flutter at Mach 0.80 increases to near $6 \mathrm{~Hz}$, while at other Mach numbers the frequency remains close to $5 \mathrm{~Hz}$. 


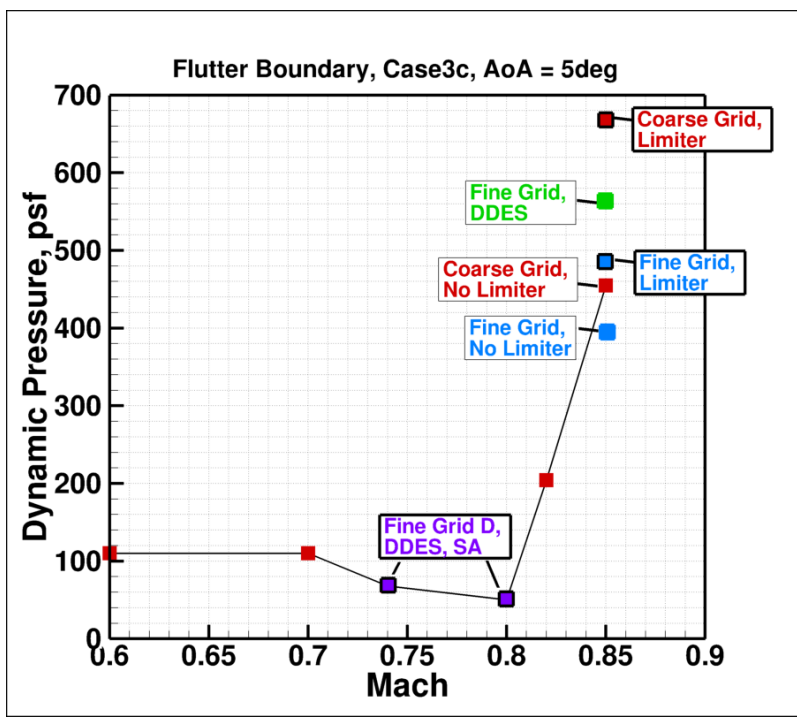

(a) Flutter Boundary Results from AePW-2. ${ }^{2}$

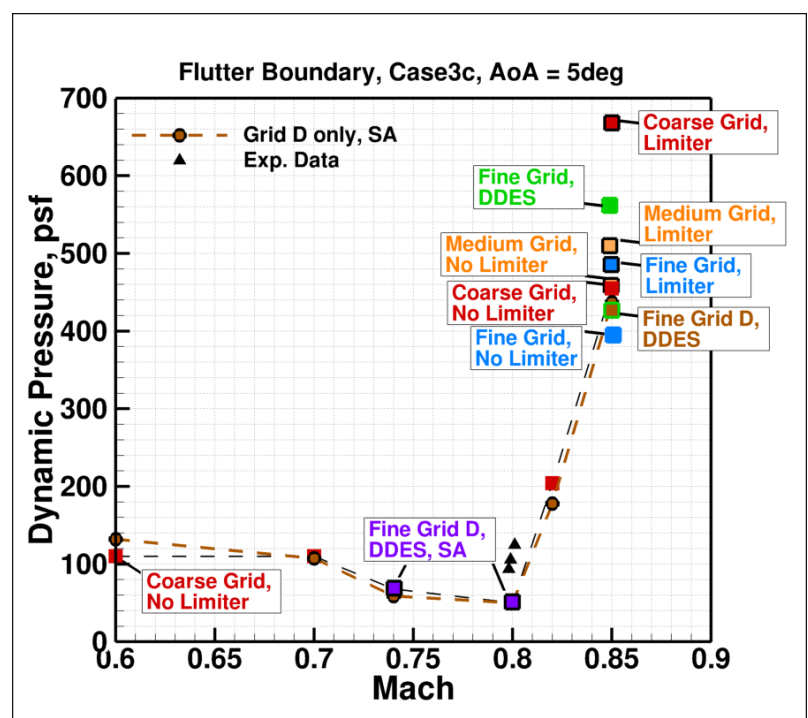

(b) Flutter Boundary Prediction with Addition of Medium Grid and Grid D Solutions.

Figure 10. Flutter Boundary Assessment, BSCW Case \#3c, Mach 0.6 - 0.85, $\alpha=5^{\circ}$.

\section{Concluding Remarks}

Reynolds-Averaged Navier-Stokes follow-up flutter prediction analyses were performed as part of AePW-2 using the NASA Langley FUN3D software. The analyses included identifying (1) the effects of the temporal and spatial convergence, (2) coupling scheme performance between fluid and structural solvers, and (3) the initial excitation and damping-input effects.

The computationally-obtained flutter dynamic pressure is $10 \%$ below the experimental flutter point for Case \#2 at Mach 0.74 and the spatial resolution only marginally affects the flutter prediction. On the other hand, selection of the time-step size and number of subiterations is especially important in the flutter boundary prediction for this case. For the Case \#3c at Mach 0.85, there is a wide range of predicted flutter points based on the spatial resolution. This varied prediction occurs because it is very difficult to computationally predict shock-induced separated flow for supercritical airfoils. The detailed temporal resolution analysis was not conducted for this case, but the selection of the time-step size and number of subiterations is based on the temporal resolution results for Case \#2.

The choice of the coupling scheme between fluid and structural solvers did not affect the prediction of the flutter boundary. However, the coupling schemes were only tested at the Mach 0.74 flow condition. Finally, the initial excitation and damping-input effects were insignificant at the Mach 0.74 condition but need to be tested at the Mach 0.85 condition. 


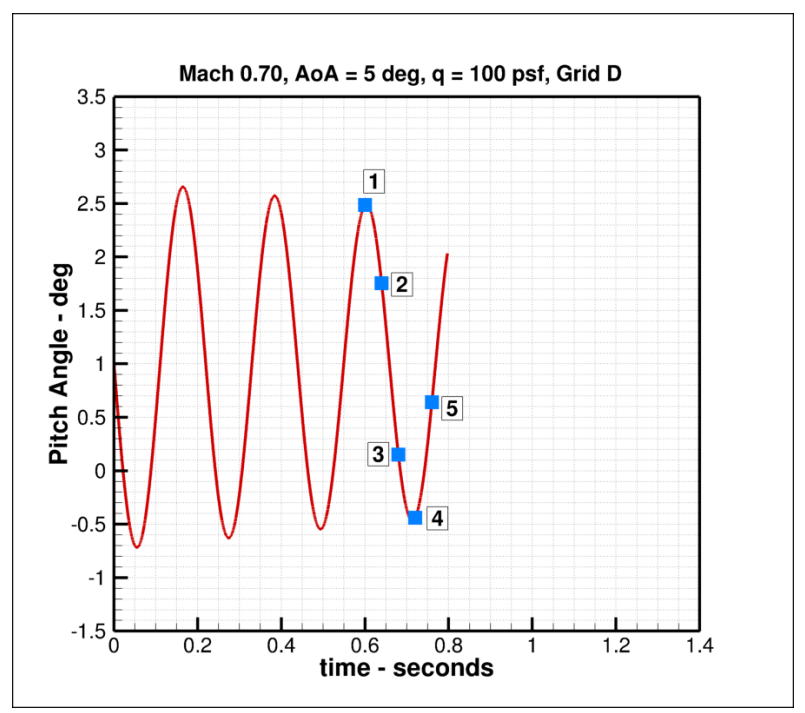

(a) Time History of Pitch Angle.

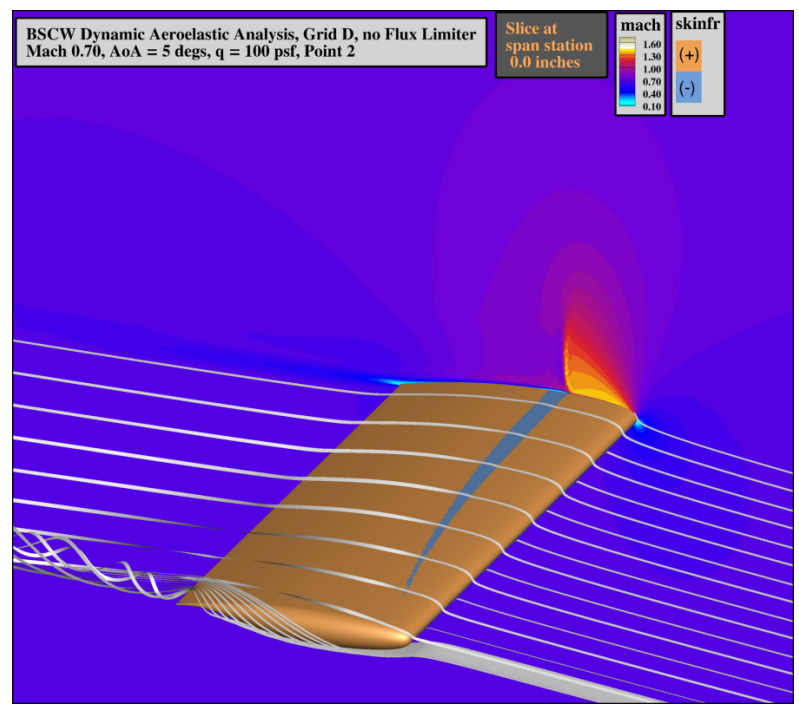

(c) Point 2.

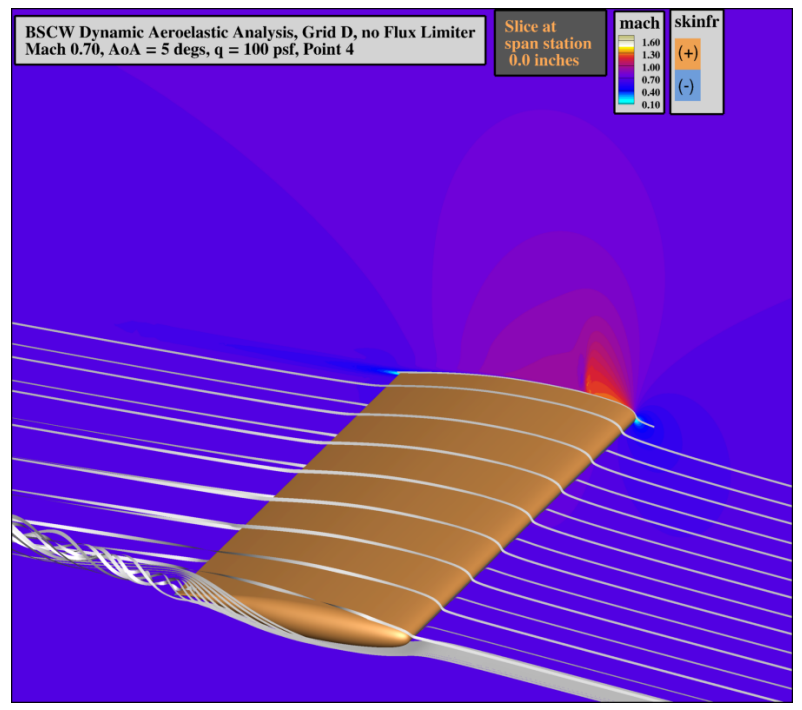

(e) Point 4 .

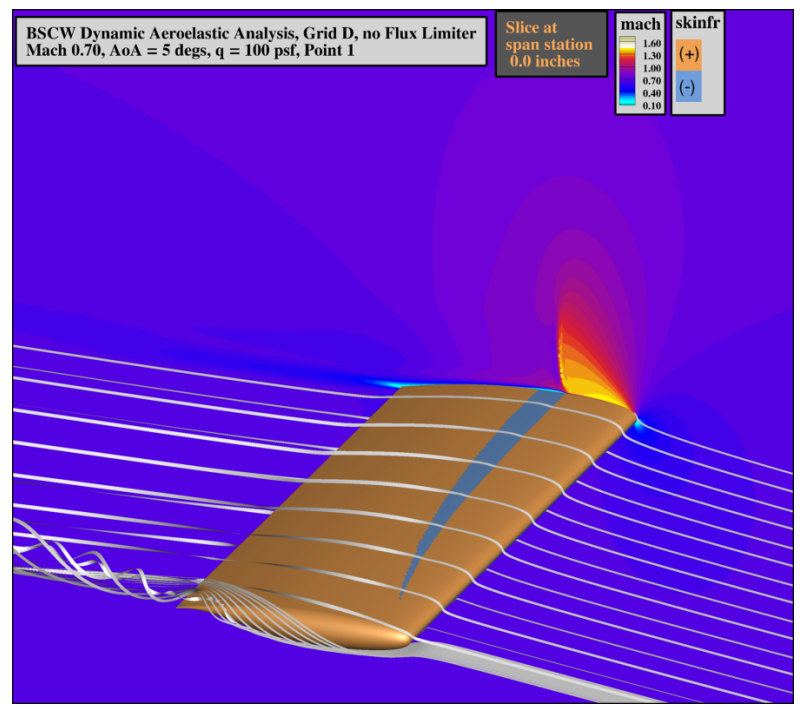

(b) Point 1.

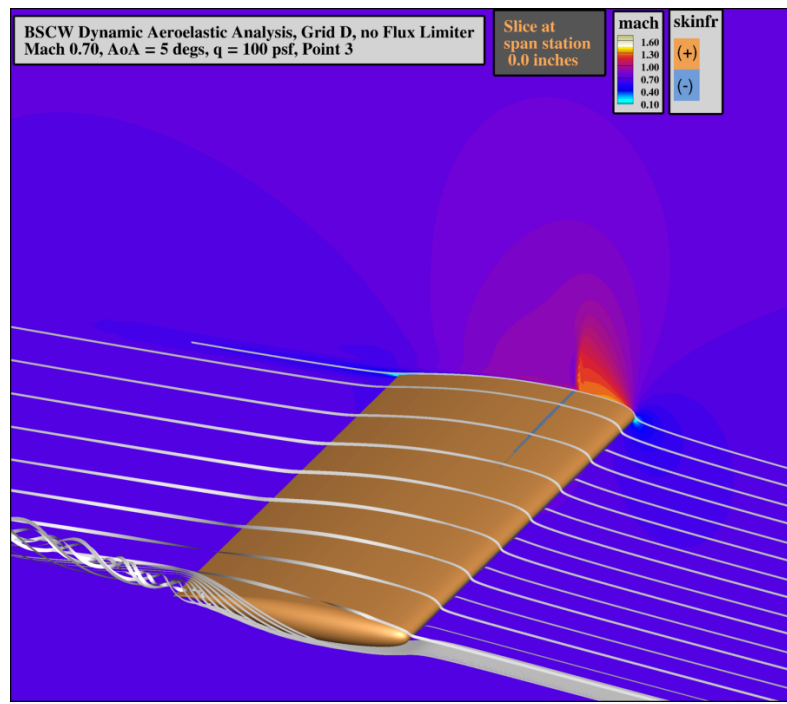

(d) Point 3.

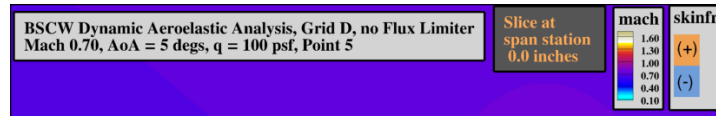

(f) Point 5

Figure 11. Flow Assessment at Mach 0.70, $\alpha=5^{\circ}, q=100$ psf.

13 of 20 


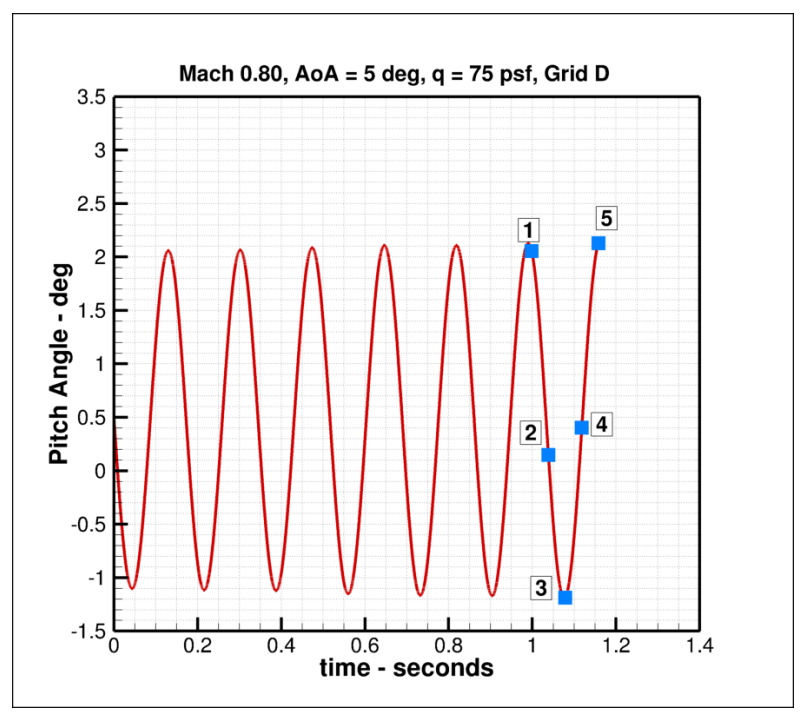

(a) Time History of Pitch Angle.

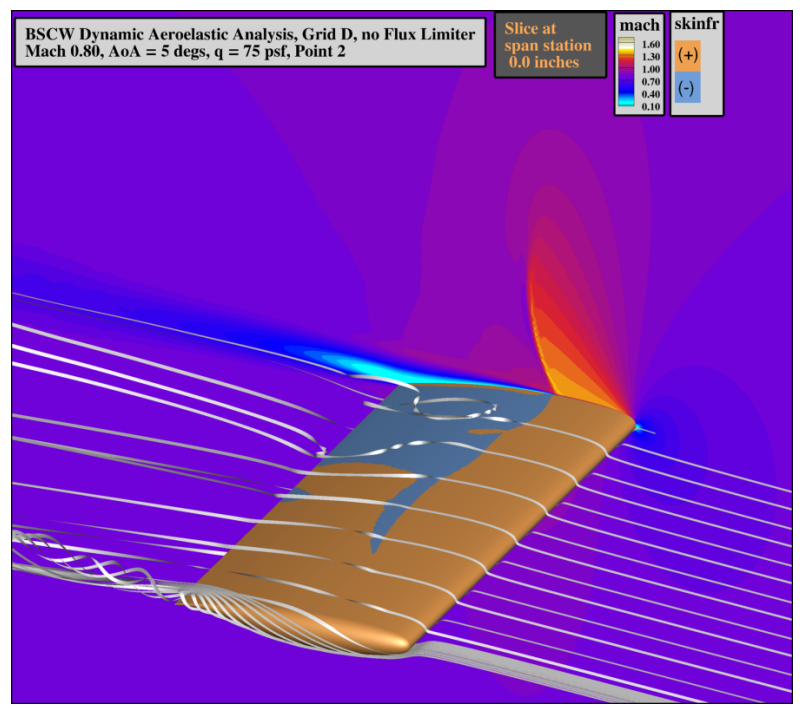

(c) Point 2.

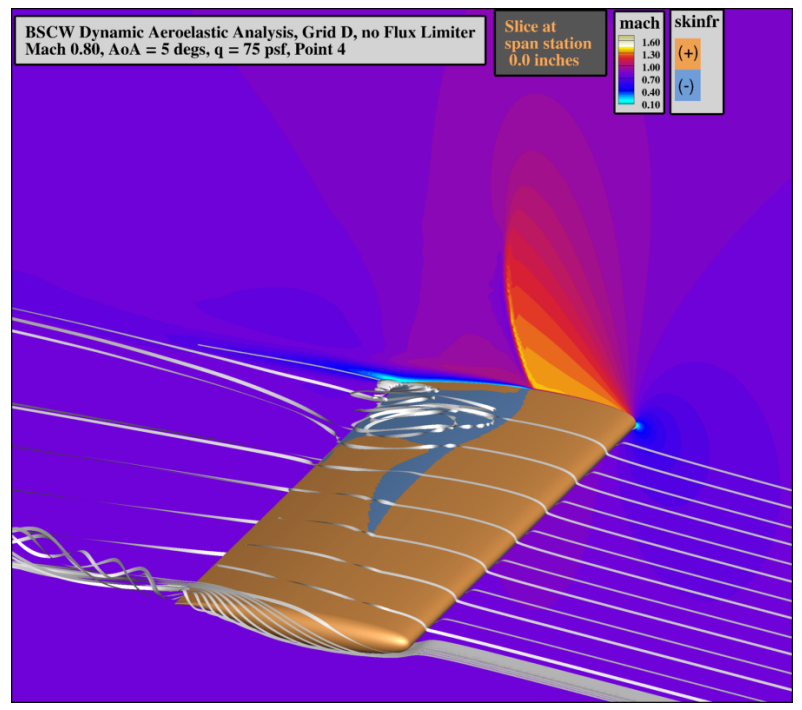

(e) Point 4 .

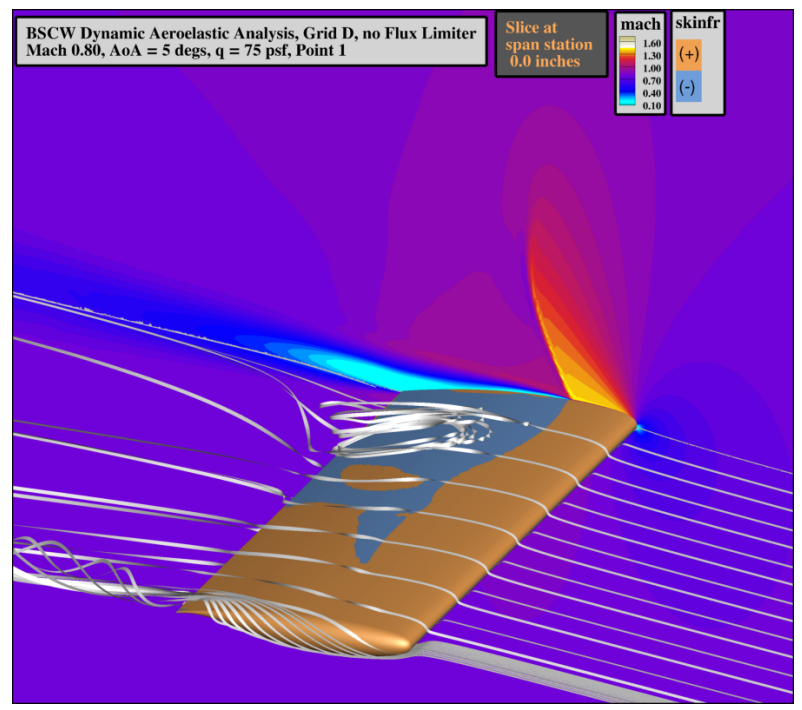

(b) Point 1.

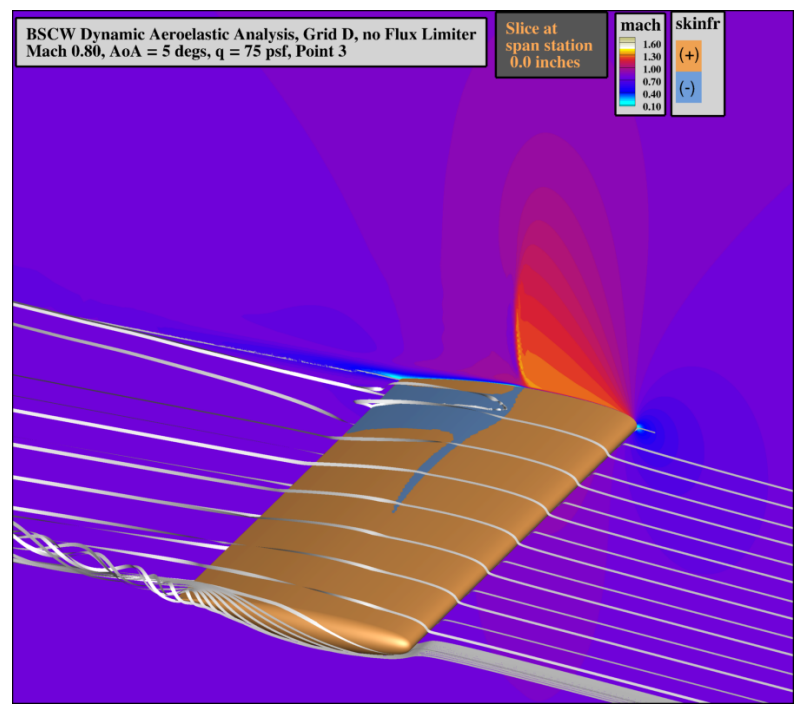

(d) Point 3.

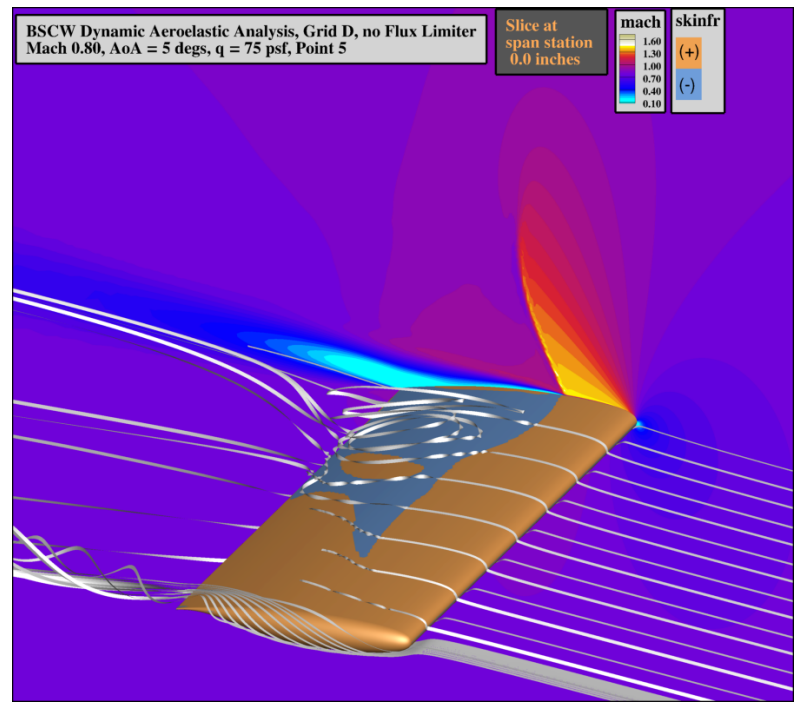

(f) Point 5.

Figure 12. Flow Assessment at Mach 0.80, $\alpha=5^{\circ}, \mathbf{q}=\mathbf{5 0}$ psf.

14 of 20 


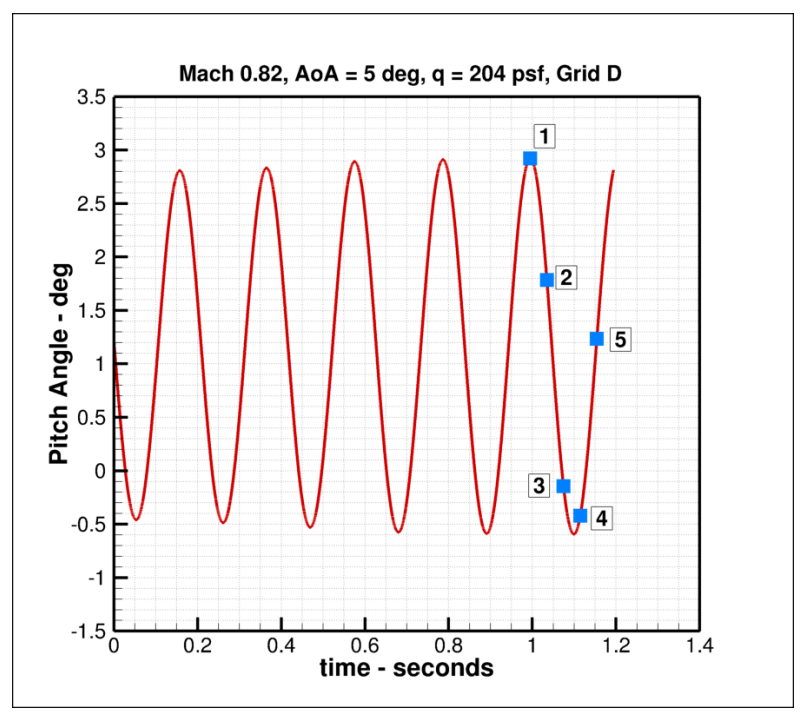

(a) Time History of Pitch Angle.

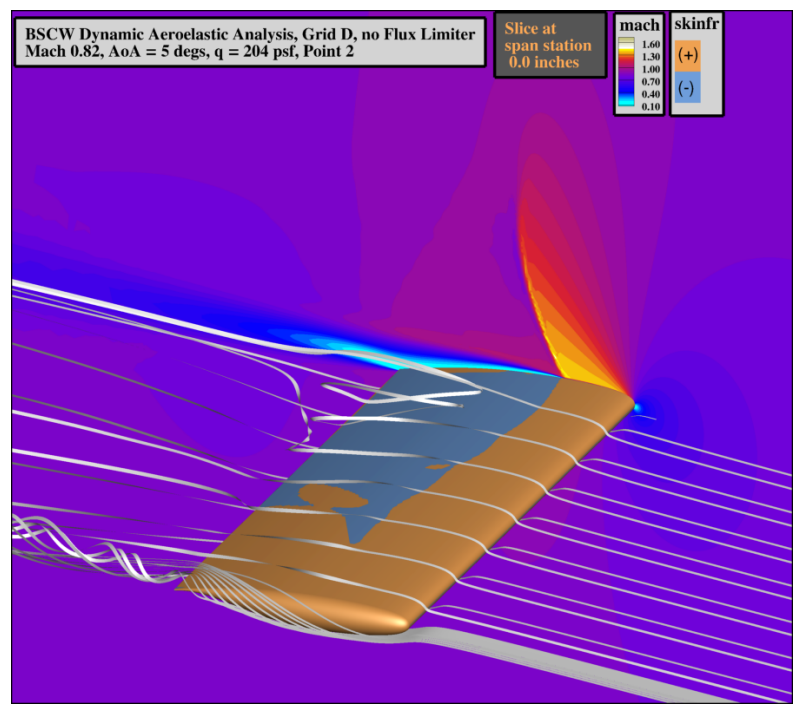

(c) Point 2.

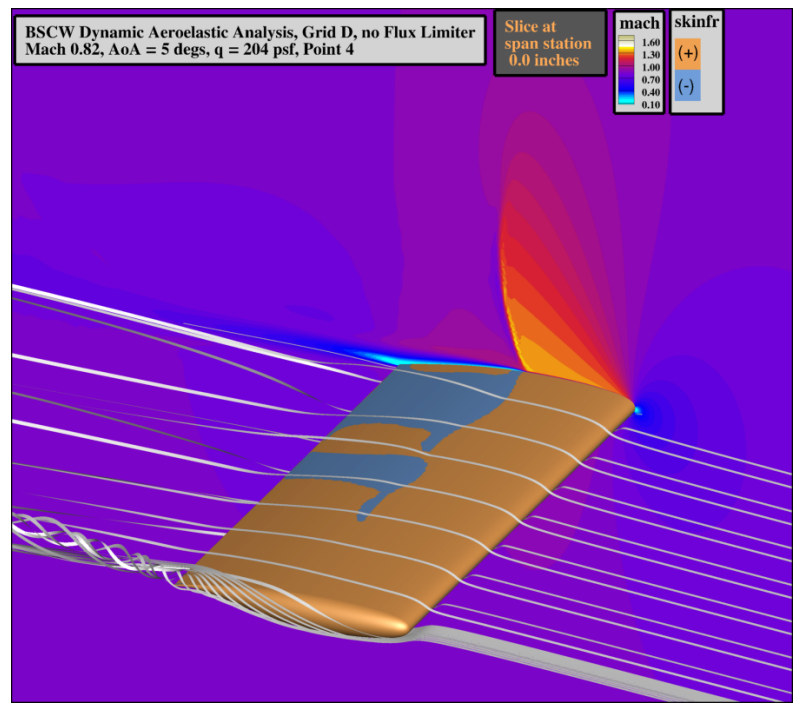

(e) Point 4 .

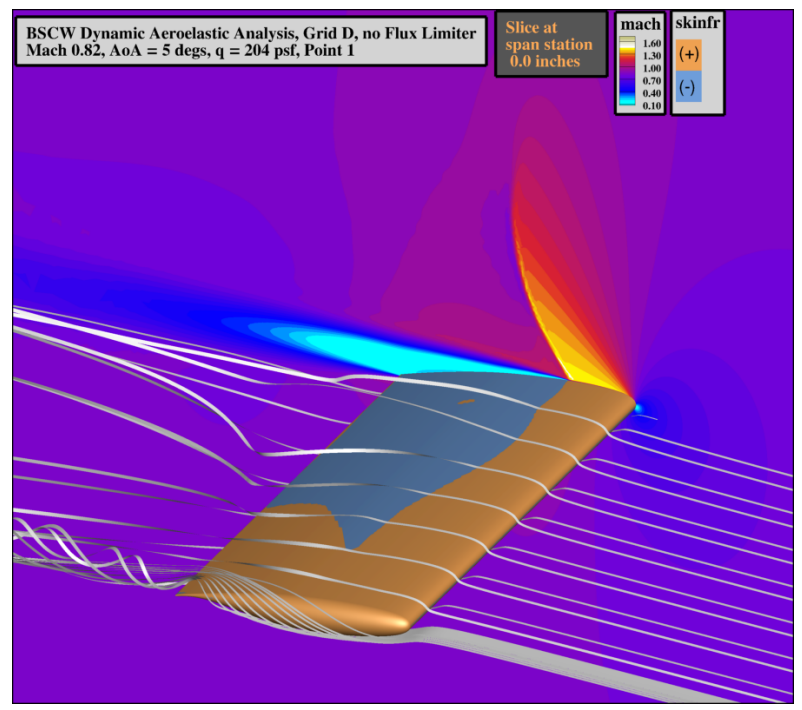

(b) Point 1.

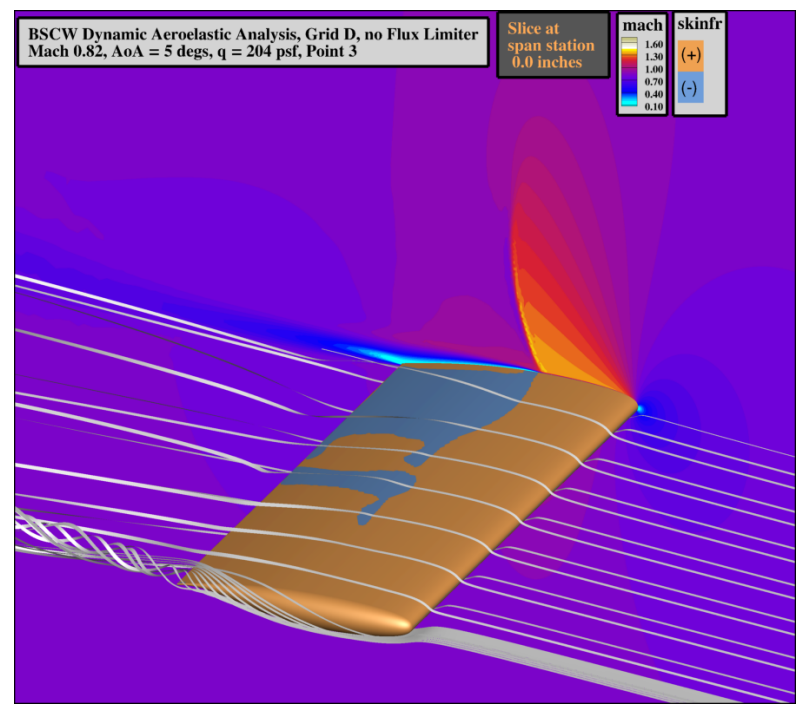

(d) Point 3.

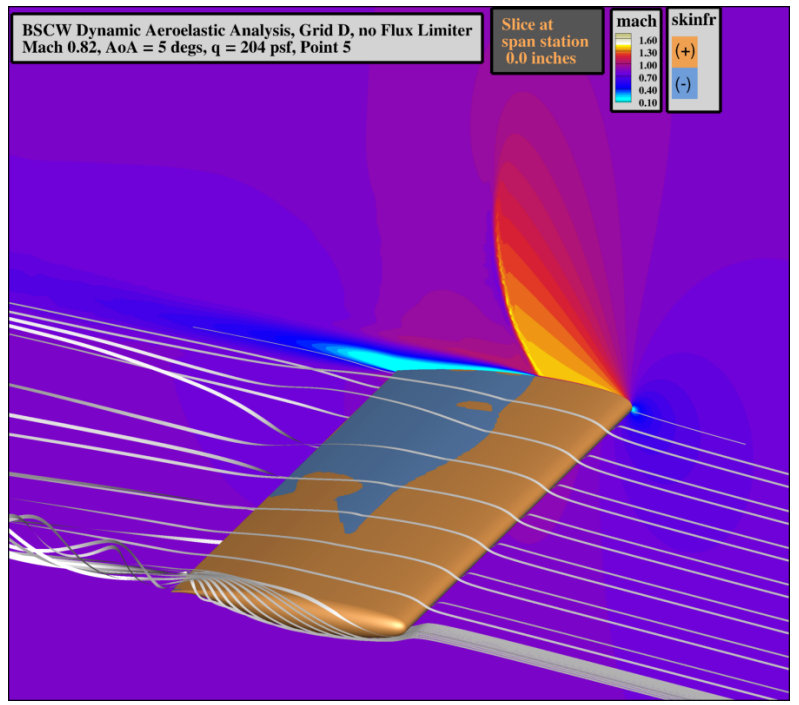

(f) Point 5.

Figure 13. Flow Assessment at Mach 0.82, $\alpha=5^{\circ}, q=204$ psf.

15 of 20 


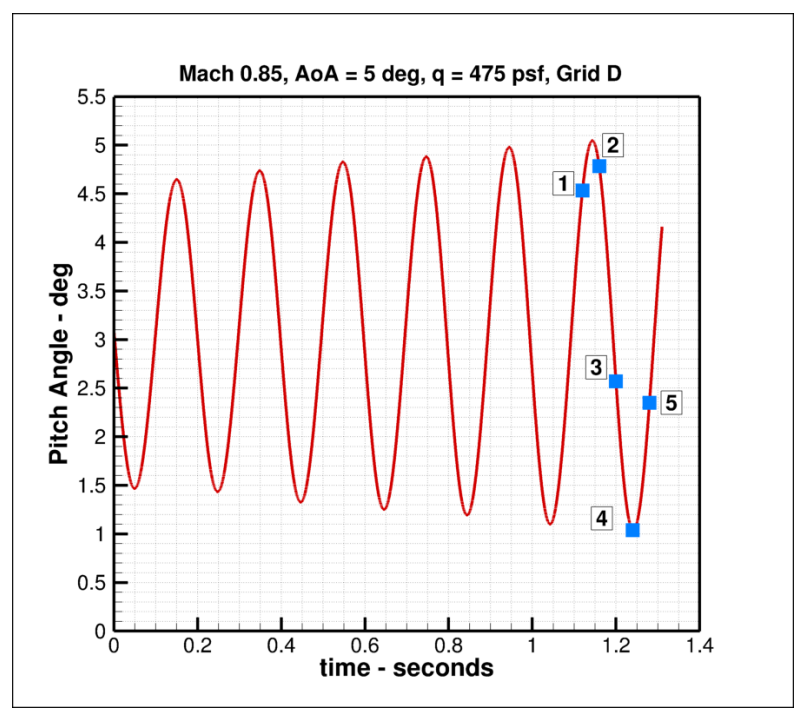

(a) Time History of Pitch Angle.

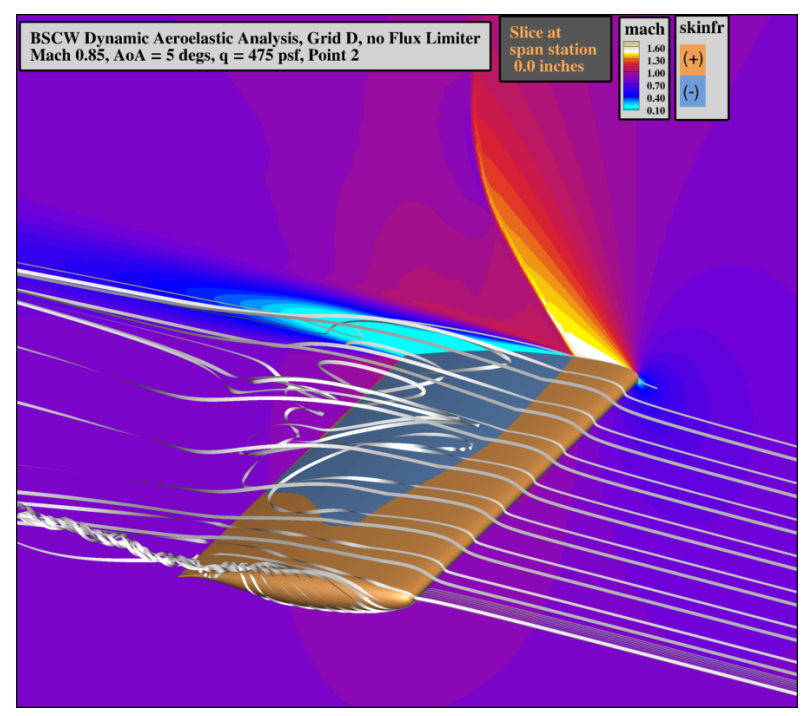

(c) Point 2.

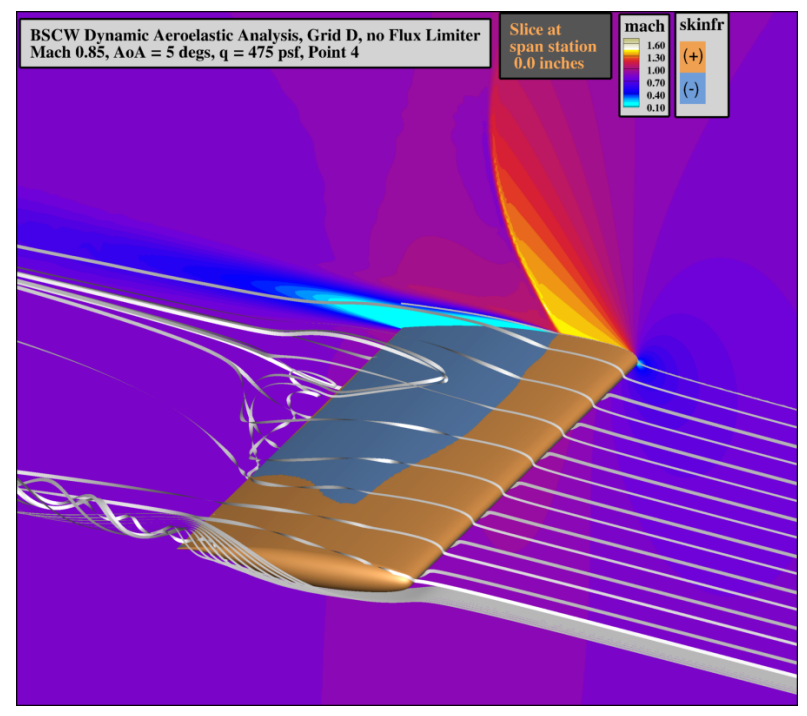

(e) Point 4 .

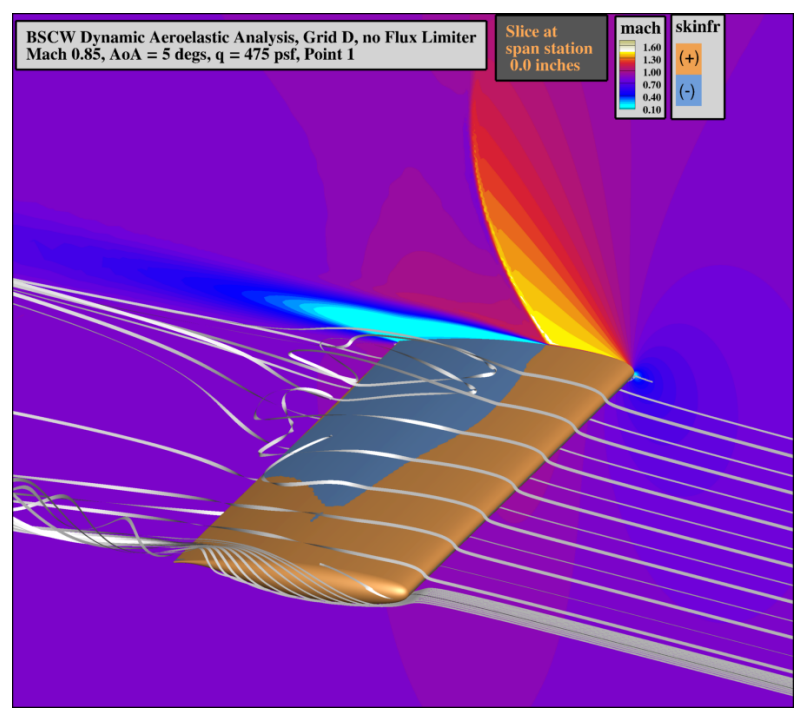

(b) Point 1.

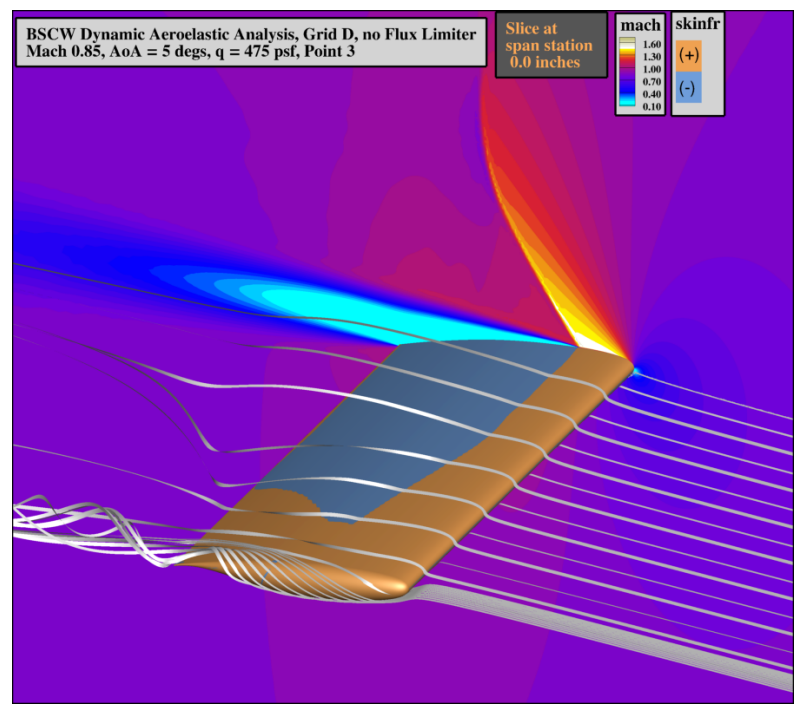

(d) Point 3.

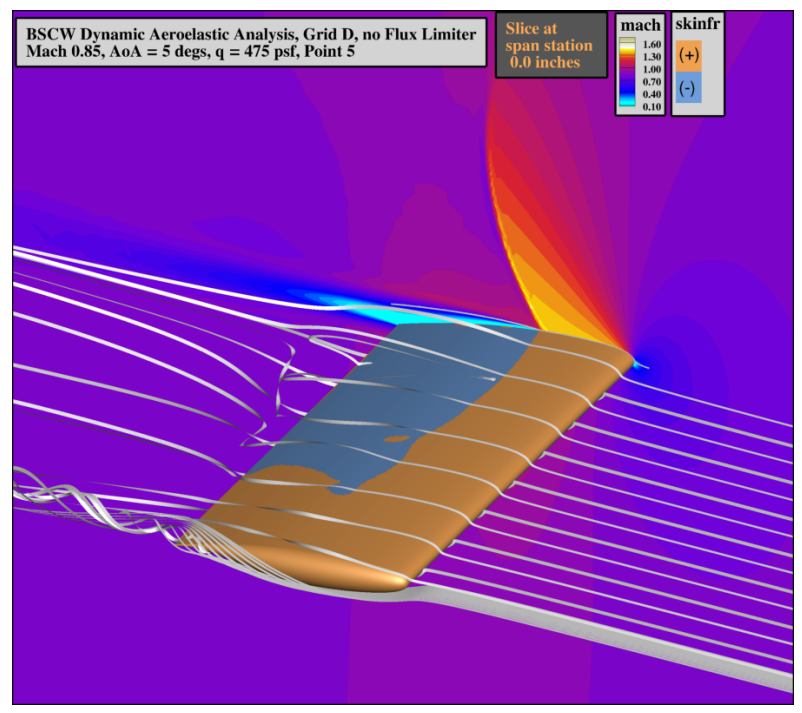

(f) Point 5.

Figure 14. Flow Assessment at Mach 0.85, $\alpha=5^{\circ}, q=475$ psf.

16 of 20 


\section{Appendix: Structural Dynamics}

The coupled linear structural dynamics equations can be written as

$$
[\mathbf{M}] \ddot{\delta}+[\mathbf{D}] \dot{\delta}+[\mathbf{K}] \delta=\mathbf{F}_{a}
$$

where $[\mathbf{M}]$ is the mass matrix, $[\mathbf{D}]$ the damping matrix, $[\mathbf{K}]$ the stiffness matrix, $\delta(\mathbf{x}, t)$ the displacement, and $\mathbf{F}_{a}(t)$ the loading, here assumed to be from aerodynamic forces only. The mass and stiffness matrices are diagonal matrices. In this work, it is assumed that the damping matrix is also diagonal. The displacements are written as an expansion in terms of natural vibration modes $\left\{\phi_{i}(\mathbf{x})\right\}$

$$
\delta=\sum_{i=1}^{N_{\text {modes }}} \mathbf{q}_{i}(t) \phi_{i}(\mathbf{x})
$$

where the coefficients of the series, $\left\{\mathbf{q}_{i}\right\}$, are referred to as the generalized coordinates. The vibration modes have associated natural frequencies $\left\{\omega_{i}\right\}$ and are orthonormalized, so that $\phi_{i}^{T} \phi_{i}=[\mathbf{I}]$. Substitution of the series representation into Equation 1 and multiplying by $\phi^{T}$ yields

$$
[\mathbf{M}] \ddot{\mathbf{q}}+[\mathbf{D}] \dot{\mathbf{q}}+[\mathbf{K}] \mathbf{q}=\phi^{T} \mathbf{F}_{a}=\hat{\mathbf{F}}_{a}
$$

where $\hat{\mathbf{F}}_{a}$ is the generalized aerodynamic force. The system represented by Equation 2 can be added to the identity system of equations $[\mathbf{I}] \dot{\mathbf{q}}+[\mathbf{0}] \ddot{\mathbf{q}}+[\mathbf{0}] \mathbf{q}-[\mathbf{I}] \dot{\mathbf{q}}=[\mathbf{0}]$, and the second-derivatives may be converted to first derivatives through the substitution $\mathbf{x}=(\mathbf{q}, \dot{\mathbf{q}})^{T}$ to give the system

$$
\left[\begin{array}{cc}
\mathbf{I} & \mathbf{0} \\
\mathbf{0} & \mathbf{M}
\end{array}\right] \dot{\mathbf{x}}+\left[\begin{array}{cc}
\mathbf{0} & -\mathbf{I} \\
\mathbf{K} & \mathbf{D}
\end{array}\right] \mathbf{x}=\left[\begin{array}{c}
0 \\
\phi^{T} \mathbf{F}_{a}
\end{array}\right]=\mathbf{u}(\mathbf{t})
$$

This may be cast as a system of first-order ordinary differential equations, one for each mode, subject to timedependent forcing terms

$$
\dot{\mathbf{x}}+\mathbf{A x}=\mathbf{B u}(\mathbf{t})
$$

where

$$
\mathbf{A}=\left[\begin{array}{cc}
\mathbf{0} & -\mathbf{I} \\
\mathbf{M}^{-1} \mathbf{K} & \mathbf{M}^{-\mathbf{1}} \mathbf{D}
\end{array}\right], \quad \mathbf{B}=\left[\begin{array}{cc}
\mathbf{I} & \mathbf{0} \\
\mathbf{0} & \mathbf{M}^{-\mathbf{1}}
\end{array}\right]
$$

In the following section, we explore two methods for advancing Equation 3 in time.

\section{A. Predictor-Corrector Scheme}

The following derives largely from Reference 21 , but it is worth repeating here to illustrate differences with the Backward-Difference Scheme described below. The exact solution to Equation 3 is

$$
\mathbf{x}=e^{-\mathbf{A} t} \int_{0}^{t} e^{\mathbf{A} \tau^{*}} \mathbf{B u}\left(\tau^{*}\right) d \tau^{*}+\text { constant }
$$

The integral in Equation 5 may be divided into arbitrary intervals in time. Evaluating the integral from $t=n \Delta t$ to $t=(n+1) \Delta t$, then making a variable substitution $\tau=\tau^{*}-n \Delta t$ yields

$$
\mathbf{x}((n+1) \Delta t)=e^{-\mathbf{A} \Delta t} \mathbf{x}(n \Delta t)+\mathbf{B} \int_{0}^{\Delta t} e^{-\mathbf{A}(\Delta t-\tau)} \mathbf{u}(\tau+n \Delta t) d \tau
$$

Note that Equation 6 is exact in the interval. $\mathbf{A}$ and $\mathbf{B}$ are constant matrices and are known from structural properties. However, the integral cannot be evaluated exactly since $\mathbf{u}(\mathbf{t})$ is not known, as it reflects the aerodynamic forces acting during the interval. If, however, it is assumed that a constant, average value of the forcing function acts over the (small) integration interval, then $\mathbf{u} \approx 1 / 2\left(\mathbf{u}_{n}+\mathbf{u}_{n+1}\right)$, where $\mathbf{u}_{n}=\mathbf{u}(n \Delta t)$. Since $\mathbf{u}_{n+1}$ is not known, it is estimated in a predictor step as $\mathbf{u}_{n+1} \approx \mathbf{u}_{n}+\left(\mathbf{u}_{n}-\mathbf{u}_{n-1}\right)$. The forcing function is now comprised of known (constant) values at a previous time interval, and may be extracted from the integrand to give

$$
\mathbf{x}_{n+1}=e^{-\mathbf{A} \Delta t} \mathbf{x}_{n}+\frac{1}{2}\left(3 \mathbf{u}_{n}-\mathbf{u}_{n-1}\right) \mathbf{B} \int_{0}^{\Delta t} e^{-\mathbf{A}(\Delta t-\tau)} d \tau
$$


The state-transition matrix

$$
\Phi(\Delta t)=e^{-\mathbf{A} \Delta t}
$$

and the integral of the state-transition matrix

$$
\Theta(\Delta t)=\int_{0}^{\Delta t} e^{-\mathbf{A}(\Delta t-\tau)} d \tau
$$

are constants and may be evaluated exactly using, for example, Laplace transforms. Thus, the predictor step is

$$
\text { predictor : } \mathbf{x}_{n+1, m=1}=\Phi \mathbf{x}_{n}+\frac{1}{2} \Theta \mathbf{B}\left(3 \mathbf{u}_{n}-\mathbf{u}_{n-1}\right)
$$

where $m$ is a subiteration counter that denotes an intermediate solution. Once $\mathbf{x}_{n+1, m=1}$ is available, an improved estimate for $\mathbf{u}_{n+1, m=2}$ may be computed, and this new estimate may be used with $\mathbf{u} \approx 1 / 2\left(\mathbf{u}_{n}+\mathbf{u}_{n+1}\right)$ in Equation 6 to give the corrector step

$$
\text { corrector : } \mathbf{x}_{n+1, m=2}=\Phi \mathbf{x}_{n}+\frac{1}{2} \Theta \mathbf{B}\left(\mathbf{u}_{n}+\mathbf{u}_{n+1}\right)
$$

\section{B. Backward Differentiation Formula Scheme}

The Predictor-Corrector Scheme outlined above has been used successfully in a sequence of NASA-developed CFD codes, beginning in the 1980s with the CAP-TSD transonic small disturbance solver, ${ }^{14}$ then in the CFL3D NavierStokes block-structured solver, ${ }^{15}$ and most recently in the FUN3D unstructured solver. ${ }^{13}$ In the CFL3D and FUN3D CFD codes, the flow equations are advanced using one of several Backward-Difference Schemes (typically secondorder in time). Historically, the coupling between the flow solver and the structures solver has been one way: within a time step, the flow equations were solved first, then the structural-dynamics equations were solved, with the corrector bringing in the latest flow solution in the time step. Within a step, the flow equations were never solved again using the latest structural solution. The Backward-Difference Scheme developed below was implemented to unify the time advancement for both fluid and structures, and potentially provide a tighter coupling between the two. The resulting method is similar to the one described in Reference 22.

Writing the time derivative at time step $n+1$ in Equation 3 as a sequence of solution states at different levels in time, weighted with appropriate coefficients, gives

$$
\dot{\mathbf{x}}_{n+1}=\frac{1}{\Delta t}\left[v_{n+1} \mathbf{x}_{n+1}+v_{n} \mathbf{x}_{n}+v_{n-1} \mathbf{x}_{n-1}+v_{n-2} \mathbf{x}_{n-2}+\ldots\right]
$$

The sequence $\left\{v_{n}\right\}$ defines a family of backward difference formulae (BDF). The particular choice of $\left\{v_{n}\right\}$ governs the accuracy of the temporal discretization; several choices are described in Reference 23. For example, choosing $v_{n+1}=3 / 2, v_{n}=-2, v_{n-1}=1 / 2, v_{n-2}=0$ leads to a second-order accurate scheme. Substituting into Equation 3 and rearranging gives

$$
\left[\frac{v_{n+1}}{\Delta t} \mathbf{I}+\mathbf{A}\right] \mathbf{x}_{n+1}=\mathbf{B} \mathbf{u}_{n+1}-\frac{1}{\Delta t}\left[v_{n} \mathbf{x}_{n}+v_{n-1} \mathbf{x}_{n-1}+v_{n-2} \mathbf{x}_{n-2}+\ldots\right]
$$

Following the treatment of the flow equations, within the time step between $t=n \Delta t$ and $t=(n+1) \Delta t$, introduce subiterations in time, with counter $m$, such that $\mathbf{x}_{n+1, m+1} \rightarrow \mathbf{x}_{n+1}$ as $m \rightarrow \infty$. Subtracting $\left[v_{n+1} / \Delta t \mathbf{I}+\mathbf{A}\right] \mathbf{x}_{n+1, m}$ from both sides gives

$$
\left[\frac{v_{n+1}}{\Delta t} \mathbf{I}+\mathbf{A}\right] \Delta \mathbf{x}_{n+1, m+1}=\mathbf{B} \mathbf{u}_{n+1, m+1}-\mathbf{A} \mathbf{x}_{n+1, m}-\frac{1}{\Delta t}\left[v_{n+1} \mathbf{x}_{n+1, m}+v_{n} \mathbf{x}_{n}+v_{n-1} \mathbf{x}_{n-1}+v_{n-2} \mathbf{x}_{n-2}+\ldots\right]
$$

where $\Delta \mathbf{x}_{n+1, m+1}=\mathbf{x}_{n+1, m+1}-\mathbf{x}_{n+1, m}$. Except for $\mathbf{u}_{n+1, m+1}$, all terms on the right-hand side of Equation 12 are known during the time-subiteration from $m$ to $m+1$.

The term arising from the generalized aerodynamic forces, $\mathbf{u}_{n+1, m+1}$, can in principle be linearized about the $m^{\text {th }}$ level as

$$
\mathbf{u}_{n+1, m+1} \approx \mathbf{u}_{n+1, m}+\Delta \mathbf{x}_{n+1, m+1} \frac{\partial \mathbf{u}_{n+1, m}}{\partial \mathbf{x}}
$$

which results in 


$$
\left[\frac{v_{n+1}}{\Delta t} \mathbf{I}+\mathbf{A}-\frac{\partial \mathbf{u}_{n+1, m}}{\partial \mathbf{x}}\right] \Delta \mathbf{x}_{n+1, m+1}=\mathbf{B} \mathbf{u}_{n+1, m}-\mathbf{A} \mathbf{x}_{n+1, m}-\frac{1}{\Delta t}\left[v_{n+1} \mathbf{x}_{n+1, m}+v_{n} \mathbf{x}_{n}+v_{n-1} \mathbf{x}_{n-1}+v_{n-2} \mathbf{x}_{n-2}+\ldots\right]
$$

In the current implementation, the Jacobian term $\partial \mathbf{u}_{n+1, m} / \partial \mathbf{x}$ is ignored, resulting in a scheme that is not fully coupled. Future implementations may incorporate the Jacobian. To help restore the full coupling in the current implementation, a fluid-structure iteration loop is added between the fluid solver (to obtain $\mathbf{u}$ ) and structural solver (to obtain $\mathbf{x}$ ), and the resulting update for the modal amplitudes is

$$
\left[\frac{v_{n+1}}{\Delta t} \mathbf{I}+\mathbf{A}\right] \Delta \mathbf{x}_{n+1, m+1}^{n f s i}=\mathbf{B} \mathbf{u}_{n+1, m}^{n f s i}-\mathbf{A} \mathbf{x}_{n+1, m}^{n f s i}-\frac{1}{\Delta t}\left[v_{n+1} \mathbf{x}_{n+1, m}^{n f s i}+v_{n} \mathbf{x}_{n}^{n f s i}+v_{n-1} \mathbf{x}_{n-1}^{n f s i}+v_{n-2} \mathbf{x}_{n-2}^{n f s i}+\ldots\right]
$$

where $n f s i$ is the fluid-structure iteration loop counter. Typically only a few fluid-structure iterations are required to converge at a given time step.

During the $n f s i^{\text {th }}$ fluid-structure iteration, while advancing to the $(n+1)^{t h}$ time step, for the $(m+1)^{t h}$ subiteration, all terms on the right-hand side of Equation 13 are known. The coefficient matrix on the left-hand side is a constant matrix, whose inverse may be evaluated once at the start of the computation. Thus, the subiteration update is simply

$$
\Delta \mathbf{x}_{n+1, m+1}^{n f s i}=[\mathbf{L H S}]^{-1}\left[\mathbf{R H S}_{n+1, m+1}^{n f s i}\right]
$$

Finally, it should be noted that when the Backward-Difference Scheme is used to advance the structural equations within FUN3D, the subiteration counter $m$ is common to, and governed by, the same subiteration counter used to advance the flow equations. The implication is that any maximum number of subiterations, or termination of the subiteration loop due to convergence, is based on the requirements of the flow equations and not the structural equations. However, all evidence to date suggests that the structural equations are extremely well converged when subject to the convergence requirements of the flow equations. 


\section{References}

${ }^{1}$ Heeg, J., Chwalowski, P., Schuster, D. M., Raveh, D., Jirasek, A., and Dalenbring, M., "Plans and Example Results for the 2nd AIAA Aeroelastic Prediction Workshop," AIAA Paper 2015-0437, Jan. 2015.

${ }^{2}$ Chwalowski, P. and Heeg, J., "FUN3D Analyses in Support of the Second Aeroelastic Prediction Workshop," AIAA Paper 2016-3122, June 2016.

3"http://fun3d.larc.nasa.gov/papers/FUN3D_Manual-12.7.pdf", NASA/TM-2015-218761.

${ }^{4}$ Schuster, D. M., "Aerodynamic Measurements on a Large Splitter Plate for the NASA Langley Transonic Dynamics Tunnel," NASA TM 2001-210828, March 2001.

${ }^{5}$ Pirzadeh, S. Z., “Advanced Unstructured Grid Generation for Complex Aerodynamic Applications,” AIAA Paper 2008-7178, Aug. 2008.

${ }^{6}$ Samareh, J. A., "Unstructured Grids on NURBS Surfaces," AIAA Paper 1993-3454.

7"http://nescacademy.nasa.gov/workshops/AePW2/public/".

8"http://turbmodels.larc.nasa.gov/spalart.html".

${ }^{9}$ Spalart, P. R. and Allmaras, S. R., “A One-Equation Turbulence Model for Aerodynamic Flows," La Recherche Aerospatiale, No. 1, 1994, pp 5-21.

${ }^{10}$ Venkatakrishnan, V., "Convergence to Steady State Solutions of the Euler Equations on Unstructured Grids with Limiter," Journal of Computational Physics, Vol. 118, No. 1, April 1995, pages 120-130.

${ }^{11}$ Roe, P. L., "Approximate Riemann Solvers, Parameter Vectors, and Difference Schemes," Journal of Computational Physics, Vol. 43, No. 2, October 1981, pages 357-372.

${ }^{12}$ Biedron, R. T. and Lee-Rausch, E. M., "Rotor Airloads Prediction Using Unstructured Meshes and Loose CFD/CSD Coupling," AIAA Paper 2008-7341, 2008.

${ }^{13}$ Biedron, R. T. and Thomas, J. L., "Recent Enhancements to the FUN3D Flow Solver for Moving-Mesh Applications," AIAA Paper 20091360, Jan. 2009.

${ }^{14}$ Batina, J. T., Seidel, D. A., Bland, S. R., and Bennet, R. M., "Unsteady Transonic Flow Calculations for Realistic Aircraft Configurations," AIAA Paper 1987-0850, 1987.

${ }^{15}$ Bartels, R. E., Rumsey, C. L., and Biedron, R. T., “CFL3D Version 6.4 - General Usage and Aeroelastic Analysis,” NASA TM 2006-214301, March 2006.

${ }^{16}$ MSC Software, Santa Ana, CA, MSC Nastran, 2008, http://www .mscsoftware.com/products/msc_nastran.cfm.

${ }^{17}$ Samareh, J. A., "Discrete Data Transfer Technique for Fluid-Structure Interaction,” AIAA Paper 2007-4309, June 2007.

${ }^{18}$ Vatsa, V. N., Carpenter, M. H., and Lockard, D. P., "Re-evaluation of an Optimized Second Order Backward Difference (BDF2OPT) Scheme for Unsteady Flow Applications," AIAA Paper 2010-0122, Jan. 2010.

${ }^{19}$ Raveh, D. E., Yossef, Y. M., and Levy, Y., "Flow Simulations fo the Second Aeroelastic Prediction Workshop Using the EZNSS Code," AIAA Paper 2016-3124, June 2016.

${ }^{20}$ Dansberry, B. E., Durham, M. H., Bennett, R. M., Turnock, D. L., Silva, W. A., and Rivera, J. A., "Physical Properties of the Benchmark Models Program Supercritical Wing,” NASA Technical Memorandum 4457, 1993.

${ }^{21}$ Edwards, W. J., Bennett, R. M., Whitlow, W. J., and Seidel, D. A., “Time-Marching Transonic Flutter Solutions Including Angle-of-Attack Effects," AIAA Journal, Vol. 20, No. 11, 1983, pages 899-906.

${ }^{22}$ Melville, R. B., Morton, S. A., and Rizzetta, D. P., "Implementation of a Fully-Implicit, Aeroelastic Navier-Stokes Solver," AIAA Paper 1997-2039, June 1997.

${ }^{23}$ Biedron, R. T., Vatsa, V. N., and Atkins, H. L., "Simulation of Unsteady Flows Using an Unstructured Navier-Stokes Solver on Moving and Stationary Grids," AIAA Paper 2005-5093, June 2005. 